FEHMN MMS, ECD-22

\title{
MODELS AND METHODS SUMMARY for the FEHMN APPLICATION by
}

George A. Zyvoloski Bruce A. Robinson Zora V. Dash Rerernm Lynn L. Trease sep $v$ 0

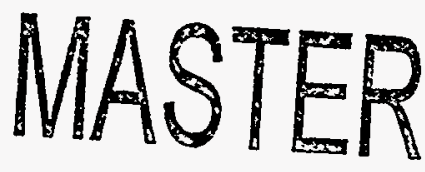


FEHMN MMS, ECD-22

LA-UR-94-3787, Rev. 1

Page 2 of 69

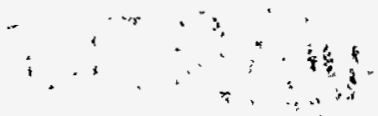




\section{DISCLAIMER}

This report was prepared as an account of work sponsored by an agency of the United States Government. Neither the United States Government nor any agency thereof, nor any of their employees, makes any warranty, express or implied, or assumes any legal liability or responsibility for the accuracy, completeness, or usefulness of any information, apparatus, product, or process disclosed, or represents that its use would not infringe privately owned rights. Reference herein to any specific commercial product, process, or service by trade name, trademark, manufacturer, or otherwise does not necessarily constitute or imply its endorsement, recommendation, or favoring by the United States Government or any agency thereof. The views and opinions of authors expressed herein do not necessarily state or reflect those of the United States Government or any agency thereof. 


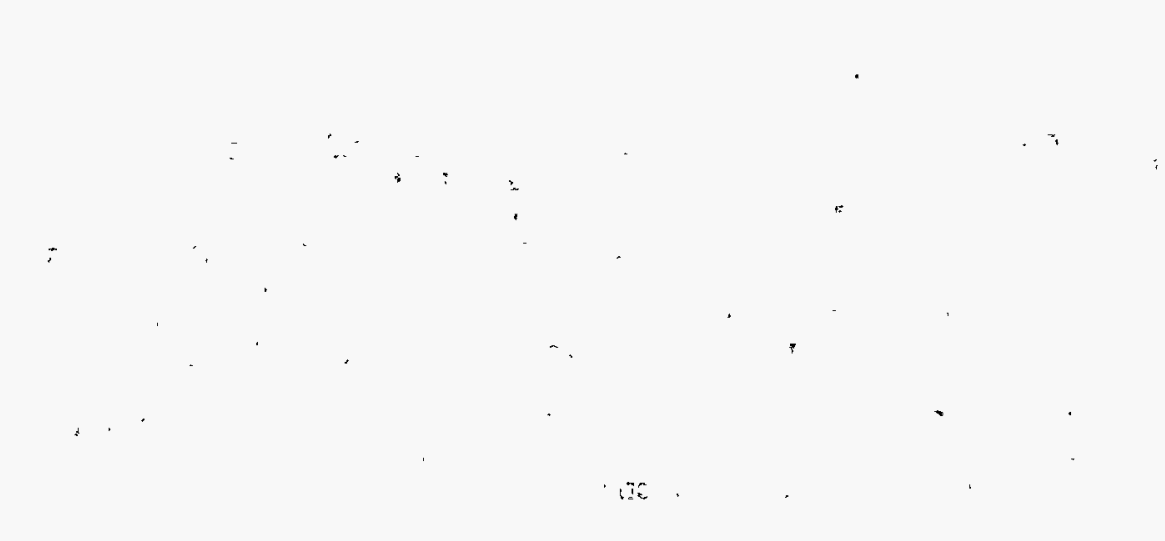




\section{DISCLAIMER}

Portions of this document may be illegible in electronic image products. Images are produced from the best available original document. 


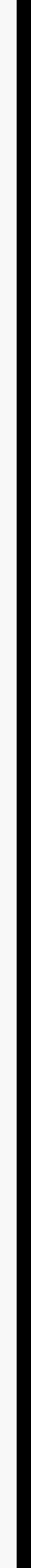




\section{TABLE OF CONTENTS}

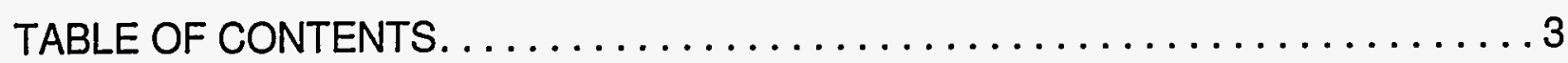

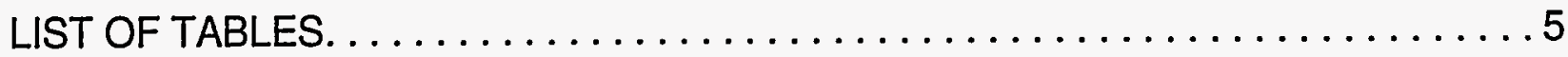

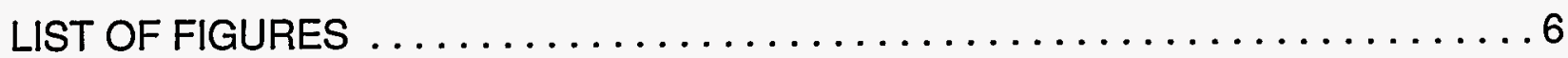

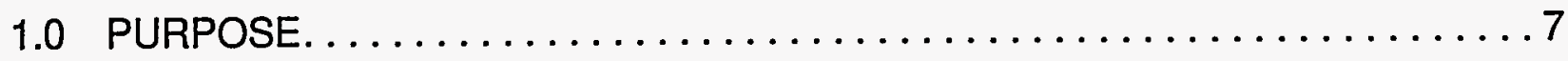

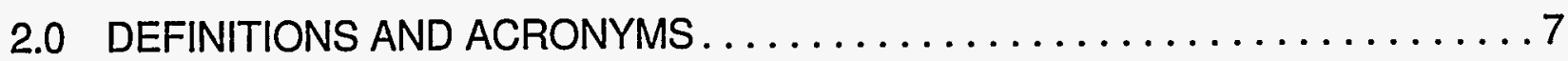

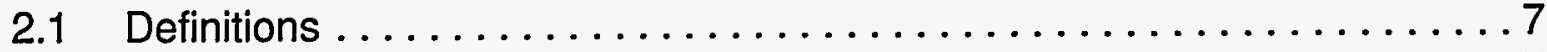

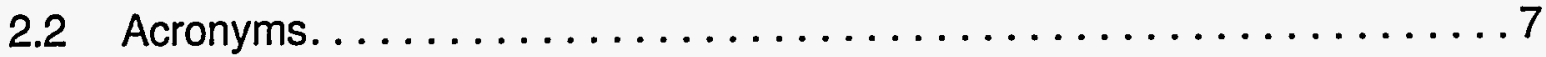

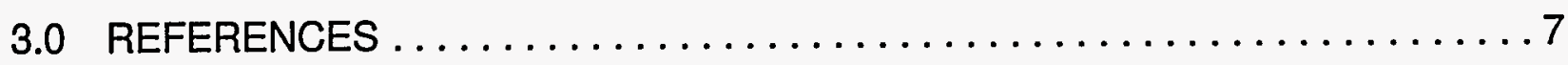

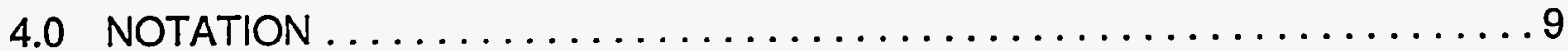

5.0 STATEMENT AND DESCRIPTION OF THE PROBLEM . . . . . . . . 16

6.0 STRUCTURE OF THE SYSTEM MODEL $\ldots \ldots \ldots \ldots \ldots \ldots \ldots \ldots \ldots$

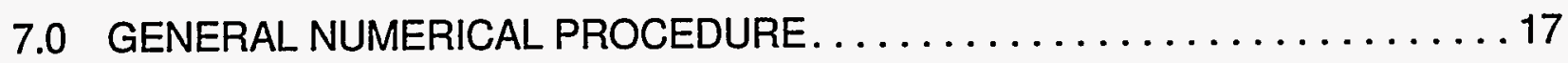

8.0 COMPONENT MODELS. . . . . . . . . . . . . . . . . . . . . 19

8.1 Flow and Energy Transport ......................

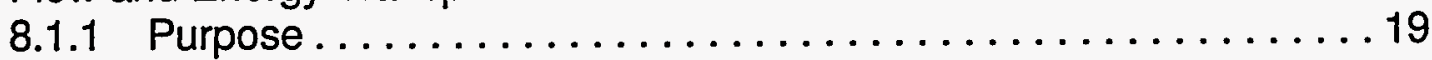

8.1.2 Assumptions and Limitations.................. 19

8.1.3 Derivation.............................. 19

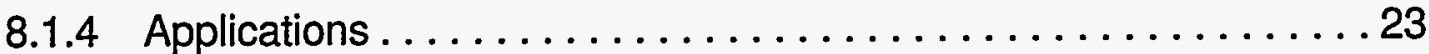

8.1 .5 Numerical Method Type........................ 24

8.1.6 Derivation of Numerical Model. . . . . . . . . . . . . . . . 24

8.1 .7 Location. . . . . . . . . . . . . . . . . . . . . . . 32

8.1.8 Numerical Stability and Accuracy ................. 32

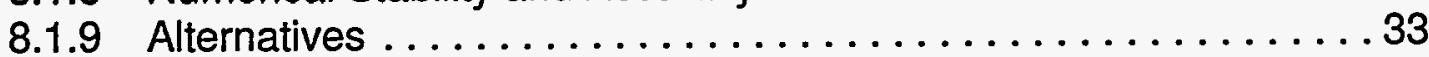

8.2 Dual Porosity and Double Porosity / Double Permeability Formulation. . . . . 33

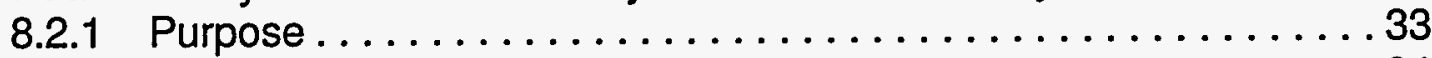

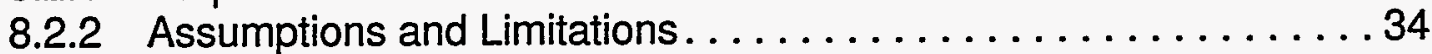

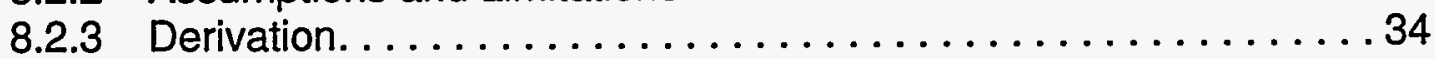

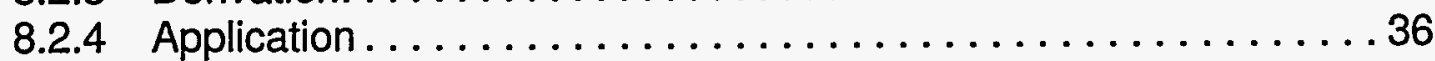

8.2.5 Numerical Method Type........................ 36

8.2.6 Derivation of Numerical Model. . . . . . . . . . . . . . . 36

8.2 .7 Location. . . . . . . . . . . . . . . . . . . . . . . 39

8.2.8 Numerical Stability and Accuracy ................... 39

8.2 .9 Alternatives .............................. 39 
8.3 Solute Transport - Reactive Transport and Particle Tracking Models . . . . 40

8.3.1 Purpose ............................ 40

8.3.2 Assumptions and Limitations................... 40

8.3.3 Derivation............................ 40

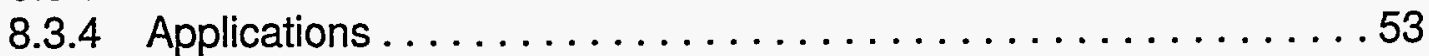

8.3.5 Numerical Method Type....................... 53

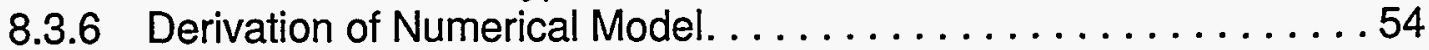

8.3.7 Location. ............................ 56

8.3.8 Numerical Stability and Accuracy ................ 56

8.3.9 Alternatives ........................... 56

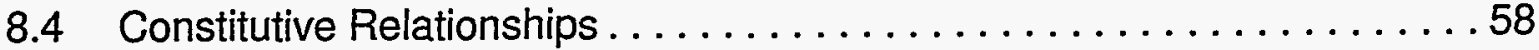

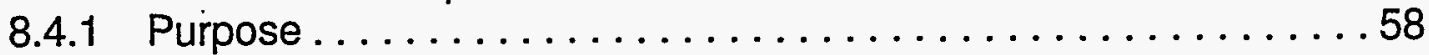

8.4 .2 Assumptions and Limitations.................. 58

8.4 .3 Derivation. . . . . . . . . . . . . . . . . . . 58

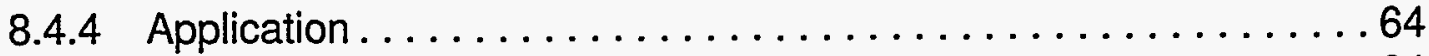

8.4 .5 Numerical Method Type......................6. 64

8.4.6 Derivation of Numerical Model. ...................64 64

8.4 .7 Location . . . . . . . . . . . . . . . . . . . . 64

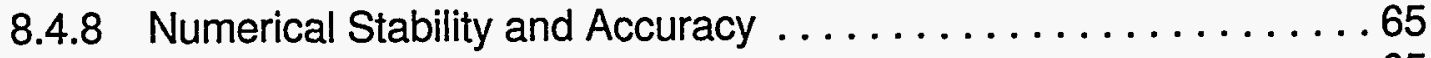

8.4 .9 Alternatives .............................. 65

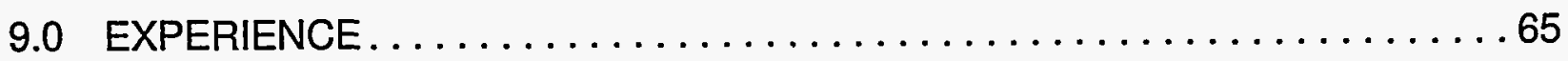

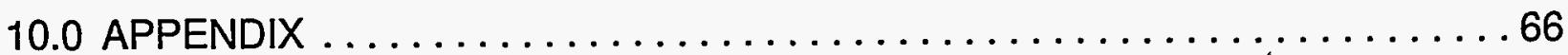




\section{LIST OF TABLES}

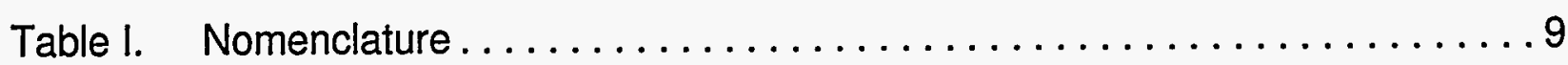

Table II. Sorption Isotherm Models. . . . . . . . . . . . . . . . 42

Table III. Polynomial Coefficients for Enthalpy Functions . . . . . . . . . . 66

Table IV. Polynomial Coefficients for Density Functions . . . . . . . . . . 67

Table V. Polynomial Coefficients for Viscosity Functions $\ldots \ldots \ldots \ldots \ldots$

Table VI. Polynomial Coefficients for Saturation Functions . . . . . . . . . . . 69 


\section{LIST OF FIGURES}

Figure 1. Simplified diagram of flow in the FEHMN application. . . . . . .

Figure 2. Comparison of nodal connections for conventional and Lobatto integrations for an orthogonal grid.

Figure 3. Area projections and internode distances used in Finite Volume calculations on a Delaunay grid.

Figure 4. Computational volume elements showing dual porosity and double porosity / double permeability parameters. .

Figure 5. Model system used to formulated the residence time transfer function for matrix diffusion 50 


\subsection{PURPOSE}

This Models and Methods Summary provides a detailed description of the mathematical models and numerical methods employed by the FEHMN application.

\subsection{DEFINITIONS AND ACRONYMS}

\subsection{Definitions}

FEHM. Finite element heat and mass transfer code (Zyvoloski, et al. 1988).

FEHMN. YMP version of FEHM (Zyvoloski, et al. 1992).

\subsection{Acronyms}

LANL. Los Alamos National Laboratory.

RTD. Residence time distribution.

RTTF. Residence time transfer function.

SOR. Simultaneous over-relaxation.

YMP. Yucca Mountain Site Characterization Project.

\subsection{REFERENCES}

Birdsell, K. H., K. Campbell, K. G. Eggert, and B. J. Travis, "Simulation of Radionuclide Retardation at Yucca Mountain Using a Stochastic Mineralogical/Geochemical Model," Proceedings of the First International Meeting on High Level Radioactive Waste Management, Las Vegas, Nevada, April 8-12 (1990).

Brigham, W. E., "Mixing Equations in Short Laboratory Cores," Soc. Pet. Eng. J. 14, 91-99 (1974).

Brownell, D. H., S. K. Garg, and J. W. Pritchett, "Computer Simulation of Geothermal Reservoirs," Paper SPE 5381, Proceedings of the 45th California Regional Meeting of the Soc. Pet. Eng. of AIME, Ventura, California (1975).

Bullivant, D. and G.A. Zyvoloski, "An Efficient Scheme for the Solution of Linear System Arising from Coupled Differential Equations," Los Alamos document, LAA-UR-903187 (1990).

Corey, A. T., "The Interrelation Between Gas and Oil Relative Permeabilities," Prod. Mon. 19, 38-41 (1954).

Dalen, V., "Simplified Finite-Element Models for Reservoir Flow Problems," Soc. Pet. Eng. J. 14, 333-343 (1974).

Friedly, J. C., and J. Rubin, "Solute Transport with Multiple Equilibrium-Controlled or Kinetically Controlled Chemical Reactions," Water Resour. Res. 28:6, 1935-1953 (1992).

Fung, L. S. K., L. Buchanan, and R. Sharma, "Hybrid-CVFE Method for Flexible -Grid Reservoir Simulation," Soc. Pet. Eng. J. 19, 188-199 (1994)

Gangi, A. F., "Variation of Whole and Fractured Porous Rock Permeability with Confining Pressure," Rock Mech. Sci. and Geomech. Abstr. 15, 249-157 (1978).

GZSOLVE Application (ECD-97). 
FEHMN MMS, ECD-22

LA-UR-94-3787, Rev. 1

Page 8 of 69

Harr, L., J. Gallagher, and G. S. Kell, NBS / NRC Steam Tables, Thermodynamics, and Transport Properties and Computer Programs for Vapor and Liquid States of Water, Hemisphere Press (1984).

Hinton, E., and D. R. J. Owen, An Introduction to Finite Element Computations, Pineridge Press, Swansea, Wales (1979).

Klavetter, E. A., and R. R. Peters, "Estimation of Hydrologic Properties of an Unsaturated Fractured Rock Mass," Sandia Report SAND84-2642 (1986).

Lu, N., "A Semianalytical Method of Path Line Computation for Transient FiniteDifference Groundwater Flow Models," Water Resour. Res. 30:8, 2449-2459 (1994).

Maloszewski, P., and A. Zuber, "On the Theory of Tracer Experiments in fissured Rocks with a Porous Matrix," J. Hydrol. 79, 333-358 (1985).

Mercer, J. W., and C. R. Faust, "Simulation of Water- and Vapor-Dominated Hydrothermal Reservoirs," Paper SPE 5520, Proceedings of the 50th Annual Fall Meeting of the Soc. Pet. Eng. of AIME, Dallas, Texas (1975).

Moench, A. F., "Double-Porosity Models for a Fissured Groundwater Reservoir with Fracture Skin," Water Resour. Res. 20:7, 831-846 (1984).

Neretnicks, I., "Diffusion in the Rock Matrix: An Important Factor in Radionuclide Migration?”, J. Geophys. Res. 85, B8, 4379-4397 (1980).

Nitao, J., "Numerical Modeling of the Thermal and Hydrological Environment Around a Nuclear Waste Package Using the Equivalent Continuum Approximation: Horizontal Emplacement," Lawrence Livermore National Laboratory Report UCID-21444(1988).

Plummer, L. N., and E. Busenberg, "The Solubilities of Calcite, Argonite, and Vaterite in $\mathrm{CO}_{2}-\mathrm{H}_{2} \mathrm{O}$ Solutions Between 0 and $90^{\circ} \mathrm{C}$, and an Evaluation of the Aqueous model for the System CaCO3-H2O," Geochim. et Cosmochim. Acta, 46, 1101 (1982).

Polzer, W. L., M. G. Rao, H. R. Fuentes, and R. J. Beckman, "Thermodynamically Derived Relationships Between the Modified Langmuir Isotherm and Experimental Parameters," submitted to Environmental Science and Technology (1992).

Pruess, K., “TOUGH Users Guide,” Lawrence Berkeley Laboratory Report LBL-20709 (June 1987).

Reeves, M. (ed), "Review and Selection of Unsaturated Flow Models", Intera Document B00000000-01425-2200-00001 Rev. 00, (1993).

Reimus, P. W., "The Use of Synthetic Colloids in Tracer Transport Experiments in Saturated Rock Fractures," Ph.D. Thesis, The University of New Mexico, Albuquerque, New Mexico (1995).

Robinson, B., "Model and Methods Summary for the SORBEQ Application," Los Alamos document SORBEQ MMS (ECD-20).

Robinson, B. A., "A Strategy for Validating a Conceptual Model for Radionuclide Migration in the Saturated Zone Beneath Yucca Mountain," Rad. Waste Manag. Envir. Rest. 19, 73-96 (1994).

Starr, R. C., R. W. Gillham, and E. A. Sudicky, "Experimental Investigation of Solute Transport in Stratified Porous Media 2. The Reactive Case," Water Resour. Res. 21:7, 1043-1050 (1985).

Sychev, V. V., et al., Thermodynamic Properties of Air, Hemisphere Publishing Corp. (1988).

Tang, D. H., E. O. Frind, and E. A. Sudicky, "Contaminant Transport in Fractured Porous Media: Analytical Solution for a Single Fracture," Water Resour. Res. 17:3, 555-564 (1981). 
Tompson, A. F. B., and L. W. Gelhar, "Numerical simulation of Solute Transport in Three-Dimensional, Randomly Heterogeneous Porous Media," Water Resour. Res., 26, 10, 2541-2562 (1990).

van Genuchten, M. T., "A Closed Form Equation for Predicting Hydraulic Conductivity of Unsaturated Soils," Soil Sci. Soc. Am. J. 44, 892-898 (1980).

Warren, J. E., and P. J. Root, "The Behavior of Naturally Fractured Reservoirs," Soc. Pet. Eng. J. 3 245-255 (1963).

Weeks, E. P., "Effect of Topography on Gas Flow in Unsaturated Fractured Rock: Concepts and Observations," Proceedings of the American Geophysical Union Symposium on Flow and Transport in Unsaturated Fractured Rock, D. Evans and T. Nicholson, Eds., Geophysical Monograph 42, AGU (1987).

Yeh, G. T., and V. S. Tripathi, "A Critical Evaluation of Recent Developments in Hydrogeochemical Transport Models of Reactive Multichemical Components," Water Resour. Res. 25, 93-108 (1989).

Young, L. C., "A Finite Element Method for Reservoir Simulation," Soc. Pet. Eng. J. 21, 115-128 (1981).

Zienkiewicz, O. C., The Finite Element Method, McGraw-Hill, London (1977).

Zienkiewicz, O. C., and C. J. Parekh, "Transient Field Problems - Two and Three

Dimensional Analysis by Isoparametric Finite Elements," Int. J. Numer. Methods Eng. 2, 61-70 (1973).

Zyvoloski, G., "Finite Element Methods for Geothermal Reservoir Simulation," Int. J. Numer. Anal. Methods Geomech. 7, 75-86 (1983).

Zyvoloski, G. A., M. J. O'Sullivan, and D. E. Krol, “Finite Difference Techniques for Modeling Geothermal Reservoirs," Int. J. Numer. Anal. Methods Geomech. 3, 355-366 (1979).

Zyvoloski, G. A., Z. V. Dash, and S. Kelkar, "FEHM: Finite Element Heat and Mass Transfer Code," LA-11224-MS (1988).

Zyvoloski, G. A., Z. V. Dash, and S. Kelkar, "FEHMN 1.0: Finite Element Heat and Mass Transfer Code," LA-12062-MS, Rev. 1 (1992).

Zyvoloski, G. A., and Z. V. Dash, "Software Verification Report FEHMN Version 1.0," LA-UR-91-609 (1991).

\subsection{NOTATION}

Variables used in derivation of the component and numerical model are enumerated in

Table I with reference to the equations in which they appear.

\section{Table I. Nomenclature}

\section{General notation conventions}

$\tilde{\mathrm{A}}$

$\overline{\mathrm{A}}$

[A]

$\{\mathrm{A}\}$

Approximation of A

Vector A

Two dimensional array A

One dimensional array/vector $\mathrm{A}$

" Units given in ML $\theta T$ system of dimensions: mass [M], length [L], time [ $\theta$ ], temperature [T] 
Table I. Nomenclature (Continued)

\section{Subscripts}

$a$

\begin{tabular}{c|l}
$a$ & $\begin{array}{l}\text { Subscript denoting air properties } \\
c\end{array}$ \\
cap & $\begin{array}{l}\text { Subscript denoting concentration } \\
\text { Subscript denoting capillary values }\end{array}$
\end{tabular}

\begin{tabular}{c|l}
$a$ & Subscript denoting air properties \\
$c$ & Subscript denoting concentration \\
cap & Subscript denoting capillary values
\end{tabular}

$e \quad$ Subscript denoting energy

$f$

flow

$i, j, k$

$l$

lr

$m$

$\max$

$\min$

$P$

$p$

$r$

$S$

$T$

$v$

$v r$

$w$

$x, y, z$

$\eta$

0

$1,2, \ldots, m$,

$m+1, \ldots, n$

Subscript denoting liquid properties

Subscript denoting residual liquid

Subscript denoting maximum value

Subscript denoting minimum value

Subscript denoting fluid phase

Subscript denoting rock properties

Subscript denoting residual vapor

Subscript denoting initial value
Subscript denoting fracture properties

Subscript denoting properties of flowing fluid

Subscripts denoting nodal position (node indices)

Subscript denoting mass or matrix property for dual porosity formulations

Subscript denoting derivative with respect to pressure

Subscript denoting derivative with respect to saturation

Subscript denoting derivative with respect to temperature

Subscript denoting vapor properties

Subscript denoting water properties

Subscripts denoting coordinate direction

Subscript denoting noncondensible gas

Subscripts denoting the specie or component (i.e., nth component)

Superscripts

$U P$

$0, k, k+1$

$n, n+1$
Superscript denoting upstream-weighted value

Superscripts denoting iteration (i.e., kth iteration)

Superscripts denoting timestep (i.e., nth timestep)

* Units given in ML $\theta T$ system of dimensions: mass [M], length [L], time [ $[\theta]$, temperature $[T]$ 


\section{Table I. Nomenclature (Continued)}

\begin{tabular}{|c|c|}
\hline \multicolumn{2}{|l|}{ Parameters } \\
\hline A & Internode area projection for finite volume calculation $\left(L^{2}\right)[$ Figure 3$]$ \\
\hline$[A]$ & Solution matrix for system of nonlinear equations [Equations (47) - (54), (64) - (72)] \\
\hline$A_{\text {for }}$ & $\begin{array}{l}\text { Arrhenius equation model parameter (frequency factor), for units see discussion of } \\
\text { control statement rxn in FEHMN.UM [Equation (85)] }\end{array}$ \\
\hline$A_{H}$ & $\begin{array}{l}\text { Henry's Law coefficient model parameter }\left[\left(\frac{M}{L \theta^{2}}\right) \text { per mole fraction of liquid }\right] \text { [Equation } \\
(80)]\end{array}$ \\
\hline$A_{H, i}, \mathrm{i}=1,5$ & Constants in temperature dependent Henry's Law expression [Equation (82)] \\
\hline$A_{c}$ & Concentration (solute) accumulation term $\left(\frac{\text { moles }}{\mathrm{L}^{3}}\right)$ [Equations $\left.(36),(75),(76)\right]$ \\
\hline$A_{e}$ & Energy accumulation term $\left(\frac{\mathrm{M}}{\mathrm{L} \theta^{2}}\right)$ [Equations (4), (5), (10), (16), (26)] \\
\hline$A_{m}$ & Mass accumulation term $\left(\frac{M}{L^{3}}\right)$ [Equations (1), (2), (9), (25)] \\
\hline$A_{r x n, i}, \mathrm{i}=1,5$ & Constants in temperature dependent equilibrium constant expression [Equation (89)] \\
\hline$A_{\eta}$ & Noncondensible gas accumulation term $\left(\frac{M}{L^{3}}\right)$ [Equations (19), (20), (27)] \\
\hline$a_{1}, a_{2}, \ldots, a_{n}$ & Stoichiometric coefficients used in reaction rate model [Equations (83), (84)] \\
\hline$B$ & Species/solute in the reaction rate equation [Equations (83), (84), (90)] \\
\hline$b$ & Exponent in the reaction rate equation [Equation (84)] \\
\hline$a$ & Air conservation variable [Equations (50), (51)] \\
\hline$\{b\}$ & $\begin{array}{l}\text { Residual vector, right hand side (forcing function) for system of linear equations } \\
\text { [Equations }(46),(64)-(72)]\end{array}$ \\
\hline$C$ & $\begin{array}{l}\text { Concentration (solute) }\left(\frac{\text { moles }}{M}\right) \text { [Equations (36), (37), (39), (73) - (76), (78), (79), } \\
\text { Table II] }\end{array}$ \\
\hline$\hat{C}$ & Normalized concentration [Equations (96)and (97)] \\
\hline$[\hat{C}]$ & Capacitance matrix [Equations (25), (26), (27), (32), (36)] \\
\hline$c$ & Compressibility $\left(\frac{L \theta^{2}}{M}\right)$ [Equation (129)] \\
\hline$c_{p}$ & Heat capacity/Specific heat $\left(\frac{L^{2}}{\theta^{2} T}\right)$ [Page 20, Equations (113), (114)] \\
\hline
\end{tabular}


Table I. Nomenclature (Continued)

\begin{tabular}{|c|c|}
\hline$D_{A B}$ & Solute diffusion coefficient $\left(\frac{L^{2}}{\theta}\right)$ [Equation (77)] \\
\hline$D_{c l}$ & Combination of molecular diffusion and dispersivity $\left(\frac{\mathrm{L}^{2}}{\theta}\right)$ [Equation (77)] \\
\hline$D_{v a}$ & Air water diffusivity $\left(\frac{\mathrm{L}^{2}}{\theta}\right)$ [Equations (20), (21), (27), (30), (35)] \\
\hline$D_{\mathrm{c}}$ & Dispersion coefficient for tracer $\left(\frac{L^{2}}{\theta}\right)$ [Equations $(36),(38),(76)$ ] \\
\hline$D_{e}$ & Energy transmissibility term $\left(\frac{\mathrm{L}^{2}}{\theta}\right)$ [Equations (10), (12), (29), (34)] \\
\hline$D_{\text {eff }}$ & Effective dispersion coefficient of a solute [Equation (93)] \\
\hline$D_{m}$ & $\begin{array}{l}\text { Mass transmissibility term }(\theta) \text { [Equations (9), (11), (12), (20), (22), (23), (28), (33), } \\
(35),(37),(39),(76)]\end{array}$ \\
\hline d & Internode distance for finite volume calculation (L) [Figure 3] \\
\hline$E$ & Young's modulus $\left(\frac{M L}{\theta^{2}}\right)$ [Equation (78)] \\
\hline$E_{\text {for }}$ & Arrhenius equation model parameter (activation energy) $\left(\frac{\mathrm{ML}^{2}}{\theta^{2} \text { moles }}\right)$ [Equation (85)] \\
\hline$\{F\}$ & Equation residuals [Equations (25), (26), (27), (36), (42) - (54)] \\
\hline$\|F\|$ & $\begin{array}{l}l^{2} \text { norm of residuals (square root of the sum of the residuals squared) [Equations (44), } \\
(45)]\end{array}$ \\
\hline$\left(\frac{\partial\{F\}}{\partial\{x\}^{k}}\right)$ & Jacobian matrix for nonlinear system [Equation (43)] \\
\hline$\overline{f_{c}}$ & Flux vector for concentration equations $\left(\frac{\text { moles }}{L^{2} \theta}\right)$ [Equation (73)] \\
\hline $\bar{f}_{e}$ & Flux vector for energy equation $\left(\frac{M}{\theta^{3}}\right)$ [Equations $\left.(4),(6)\right]$ \\
\hline$\overline{f_{m}}$ & Flux vector for mass equation $\left(\frac{M}{L^{2} \theta}\right)$ [Equations (1), (3)] \\
\hline$\overline{f_{\eta}}$ & Flux vector for noncondensible gas equation $\left(\frac{M}{L^{2} \theta}\right)$ [Equation (17)] \\
\hline$f(t)$ & Function at time $t$ [Equation (24)] \\
\hline
\end{tabular}




\section{Table I. Nomenclature (Continued)}

\begin{tabular}{|c|c|}
\hline$f^{\prime}(t)$ & Derivative of $f$ with respect to time [Equation (24)] \\
\hline$\{G\}$ & $\begin{array}{l}\text { Gravity term coefficients [Equations (25), (26), (27), (33) - (36), (39)] } \\
\text { Acceleration of gravity }\left(\frac{L}{\theta^{2}}\right) \text { [Equations (9), (10), (20), (22), (23), (25), (26), (27), (36), } \\
(76) \text { ] }\end{array}$ \\
\hline $\bar{g}$ & $g$ times the unit vector in the gravitational (z) direction [Equations (7), (8)] \\
\hline$\Delta H_{H}$ & Henry's Law equation model parameter (heat of reaction) $\left(\frac{\mathrm{ML}^{2}}{\theta^{2} \text { moles }}\right)$ [Equation (80)] \\
\hline$\Delta H_{r x n}$ & Equilibrium constant model parameter (heat of reaction) $\left(\frac{\mathrm{ML}^{2}}{\theta^{2} \text { moles }}\right)$ [Equation (87)] \\
\hline$h$ & Enthalpy $\left(\frac{L^{2}}{\theta^{2}}\right)$ [Equations (6), (12), (13), (62), (63), (113), (115), (116)] \\
\hline$I_{m}$ & Mass flow impedance $\left(\frac{\theta}{L^{2}}\right)$ [Equation (40)] \\
\hline$I_{e}$ & Heat flow impedance $\left(\frac{M}{L^{3} \theta T}\right)$ [Equation (41)] \\
\hline$K$ & Thermal conductivity $\left(\frac{\mathrm{ML}}{\mathrm{T \theta}^{3}}\right)$ [Equations (6), (16), (26), (31), Page 26] \\
\hline$K_{d}$ & Retardation coefficient (linear adsorption) [Table II] \\
\hline$K_{e q}$ & Equilibrium constant [Equations (86)-(89)] \\
\hline$K_{e q, 25}$ & Equilibrium constant at $25^{\circ} \mathrm{C}$ [Equation (87)] \\
\hline$\hat{K}_{e q}$ & Intermediate term used in equilibrium constant expression [Equations (88) and (89)] \\
\hline$k_{f a c t}$ & Multiplier to increase reaction rates to approach equilibrium behavior \\
\hline$K_{H}$ & $\begin{array}{l}\text { Henry's law constant }\left(\frac{L \theta^{2}}{M}\right) \text { [Equation (118)], }\left[\left(\frac{M}{L \theta^{2}}\right) \text { per mole fraction of liquid }\right] \\
\text { [Equation (80)] }\end{array}$ \\
\hline$\hat{K}_{H}$ & Intermediate term used in expression of Henry's law constant [Equation (82)] \\
\hline$\tilde{K}_{H}$ & Intermediate term used in expression of Henry's law constant [Equation (82)] \\
\hline$k$ & Intrinsic rock permeability $\left(L^{2}\right)$ [Equations (7), (8), (11), (61), (62), (63), (132)] \\
\hline$k_{f o r}, k_{r e v}$ & Forward and reverse reaction rate constants [Equation (84), (85)] \\
\hline$k_{R n}$ & Radioactive decay rate constant [Equation (102)] \\
\hline
\end{tabular}




\section{Table I. Nomenclature (Continued)}

\begin{tabular}{|c|c|}
\hline$L$ & Flow path length $(\mathrm{m})$ \\
\hline$L_{f}, L_{f 0}, L_{f 1}, L_{f 2}$ & $\begin{array}{l}\text { Length scales used in dual porosity and double porosity / double permeability } \\
\text { problems [Equations (56), (58), (59), (60), Figure 4] }\end{array}$ \\
\hline$M$ & Molecular weight (M) [Equation (79)] \\
\hline$M_{f}$ & Fluid mass in a cell (kg) [Equation (91)] \\
\hline$m$ & Exponent used in Gangi stress model [Equation (130)] \\
\hline$\dot{m}_{\text {out }}$ & Outlet mass flow rate from one cell to another [Equation (91)] \\
\hline$n$ & $\begin{array}{l}\text { Experimental parameter used in van Genuchten relative permeability and capillary } \\
\text { pressure models [Page 62, Page 63] }\end{array}$ \\
\hline$[N]$ & Finite element shape function [Page 24, Equations (28) - (34), (37) - (39)] \\
\hline$P$ & $\begin{array}{l}\text { Pressure }\left(\frac{M}{L \theta^{2}}\right) \text { [Equations (7) - (10), (20), (22), (23), (25), (26), (27), (36), (40), (47) } \\
-(54),(61),(62),(63),(76),(79),(104)-(107),(111),(118),(127)-(129),(131)]\end{array}$ \\
\hline$P_{c}$ & Closure stress for use in Gangi stress model (ML) [Equations (130), (131)] \\
\hline$P e$ & Peclet number for dispersion [Equations (96) and (97)] \\
\hline$q_{c}$ & Concentration source term $\left(\frac{\text { moles }}{\mathrm{L}^{3} \theta}\right)$ [Equations(36), (74), (76)] \\
\hline$q_{e}$ & Energy source term $\left(\frac{M}{L \theta^{3}}\right)$ [Equations (4), (10), (13), (16), (26), (41)] \\
\hline$q_{f m}$ & $\begin{array}{l}\text { Solute flux term from fracture to matrix in particle tracking model development } \\
\text { [Equation (99)] }\end{array}$ \\
\hline$q_{m}$ & Mass source term $\left(\frac{M}{L^{3} \theta}\right)$ [Equations (1), (9), (14), (22), (23), (25), (40)] \\
\hline$q_{\eta}$ & Noncondensible gas source term $\left(\frac{M}{L^{3} \theta}\right)$ [Equations (18), (20), (27)] \\
\hline$R$ & Universal gas constant $(8.314 \mathrm{~kJ} / \mathrm{mol}-\mathrm{K})$ [Equations (80), (85), (110)] \\
\hline$R_{f}$ & Sorption retardation factor [Equation (92)] \\
\hline$R_{p}$ & Relative permeability [Equations (7), (8), (11), (15), (121) - (126)] \\
\hline$r, r_{b}$ & Parameters used in nonlinear adsorption model (Langmuir) [Table II] \\
\hline$S$ & Saturation [Equations (2), (5), (19), (22), (23), (53) - (54), (121) - (128)] \\
\hline$T$ & $\begin{array}{l}\text { Temperature (T) [Equations (6), (16), (41), (47), (48), (50) - (52), (80), (85), (104) - } \\
(107),(111),(113),(114),(131)]\end{array}$ \\
\hline$[T]$ & Stiffness matrix [Equations (25) - (29), (36), (37)] \\
\hline
\end{tabular}




\section{Table I. Nomenclature (Continued)}

\begin{tabular}{|c|c|}
\hline$T_{f f l}, T_{f l f 2}$ & Transfer terms in dual porosity solution [Equations (59) - (63)] \\
\hline$t$ & Time $(\theta)$ [Equations (1), (4), (9), (10), (16), (20) - (27), (36), (76)] \\
\hline$u$ & Internal energy $\left(\frac{\mathrm{L}^{2}}{\theta^{2}}\right)$ [Equation (5)] \\
\hline$V$ & Integral volume [Equations (28) - (34), (37) - (39)] \\
\hline$V_{f}$ & $\begin{array}{l}\text { Volume fraction for fractures in a dual porosity and double porosity / double } \\
\text { permeability problems [Equations (55) - (58)] }\end{array}$ \\
\hline$V_{f 0}, V_{f 1}, V_{f 2}$ & $\begin{array}{l}\text { Volume fractions for the matrix volumes used in dual porosity and double porosity I } \\
\text { double permeability problems [Equations }(55)-(58) \text { ] }\end{array}$ \\
\hline$V_{T}$ & Total volume of computational cell $\left(L^{3}\right)$ [[Equation $\left.(59),(60)\right]$ \\
\hline$v$ & $\begin{array}{l}\text { Superficial velocity in one-dimensional model used in particle tracking model } \\
\text { development [Equation (93)] }\end{array}$ \\
\hline $\bar{v}$ & Velocity vector $\left(\frac{L}{\theta}\right)$ [Equations (3), (6) - (8), (17)] \\
\hline$v_{l, x}$ & Darcy velocity of liquid phase, $x$-direction [Equation (77)] \\
\hline$w$ & Weighting factor for time discretization [Equation (24)] \\
\hline$X$ & $\begin{array}{l}\text { Pressure or temperature variable in rational function approximation for saturation } \\
\text { equations [Equations }(108),(109) \text { ] }\end{array}$ \\
\hline$\{x\}$ & Solution vector, [Equations (42), (43), (46), (64) - (72)] \\
\hline$\hat{x}$ & Normalized distance along flow path [Equation (96)] \\
\hline$Y$ & Polynomial in numerator of rational function approximation [Equations (104) - (109)] \\
\hline$Z$ & Polynomial in denominator of rational function approximation [Equations (104) - (109)] \\
\hline$z$ & $\begin{array}{l}\text { Coordinate oriented in the direction of gravity [Equations (9), (10), (20), (33), (34), } \\
(39),(76) \text { ] }\end{array}$ \\
\hline$\alpha$ & Coefficient of thermal expansion $\left(\frac{1}{T}\right)$ [Equation (131)] \\
\hline$\alpha_{1}, \alpha_{2}$ & Coefficients used in sorption models [Equation (78), (90), Table II] \\
\hline$\alpha_{d}$ & Dispersivity of solute in transport calculations $(L)$ \\
\hline$\alpha_{G}$ & Experimental parameter used in van Genuchten capillary pressure model [Page 63] \\
\hline$\beta$ & Exponent used in sorption models [Equation (78), (90), Table II] \\
\hline$\gamma_{r x n}$ & $\begin{array}{l}\text { Fractional approach to equilibrium computed at an iteration in the reactive transport } \\
\text { model [Equation (91)] }\end{array}$ \\
\hline$\gamma_{t o l}$ & Fractional approach to equilibrium specified for an equilibrium reaction [Equation (91)] \\
\hline
\end{tabular}

* Units given in ML $\theta \mathrm{T}$ system of dimensions: mass [M], length [L], time [ $\theta$ ], temperature [T] 
Table I. Nomenclature (Continued)

\begin{tabular}{|c|c|}
\hline$\varepsilon$ & Tolerance taken for solution scheme [Equation (45)] \\
\hline$\eta$ & Mass fraction of air [Equations (2), (3), (9), (17) - (20), (27), (35), (115) - (119)] \\
\hline$\theta$ & Exponent used in the air/water diffusion model [Equation (21)] \\
\hline$\hat{\theta}$ & Normalized time [Equations (96)and (97)] \\
\hline$\Lambda$ & $\begin{array}{l}\text { Parameter used in nonlinear adsorption model (Freundlich, modified Freundlich) } \\
\text { [Table II] }\end{array}$ \\
\hline$\lambda$ & $\begin{array}{l}\text { Parameter used in van Genuchten relative permeability and capillary pressure models } \\
\text { [Equation (125), Page 63] }\end{array}$ \\
\hline$\mu$ & Viscosity $\left(\frac{M}{L \theta}\right)$ [Equations (7), (8), (11), (15), (61), (62), (63), (119), (120)] \\
\hline$v$ & Fractional vapor flow parameter [Equations (14), (15)] \\
\hline$\rho$ & $\begin{array}{l}\text { Density }\left(\frac{M}{L^{3}}\right) \text { [Equations (3), (5), (7) - (11), (15); (17), (19) -(23), (61), (62), (63), (76), } \\
(90),(111),(112),(117)]\end{array}$ \\
\hline$\sigma$ & In situ stress $\left(\frac{\mathrm{ML}}{\theta^{2}}\right)$ [Equation (131)] \\
\hline$\tau$ & Tortuosity factor in the air/water diffusion model [Equation (21)] \\
\hline$\tau_{\text {age }}$ & Particle age since entering the model domain [Equation (102)] \\
\hline$\tau_{f}$ & Fluid residence time in a cell (s) [Equation (91)] \\
\hline$\tau_{\text {part }}$ & Particle residence time in a cell (s) [Equation (91)] \\
\hline$\tau_{1 / 2}$ & Radioactive decay half-life \\
\hline$\phi$ & Porosity [Equations (2), (5), (19), (21) - (23), (90), (129), (130), (132)] \\
\hline$\phi_{m a t}$ & Matrix porosity in particle tracking model [Equation (99)] \\
\hline$\Omega$ & Flow domain of the model [Equations (28) - (34), (37) - (39)] \\
\hline
\end{tabular}

\subsection{STATEMENT AND DESCRIPTION OF THE PROBLEM}

Yucca Mountain is extremely complex both hydrologically and geologically. The computer codes that are used to model flow must be able to describe that complexity. For example, the flow at Yucca Mountain, in both the saturated and unsaturated zones is dominated by fracture and fault flow in many areas. With permeation to and from faults and fractures, the flow is inherently three-dimensional (3-D). Birdsell, et al. (1990) presented calculations showing the importance of 3-D flow at Yucca Mountain. Coupled heat and mass transport occurs in both the unsaturated and saturated zones. In the near field region surrounding the repository, the coupled flow effects dominate the fluid behavior. Here boiling, dryout, and condensation can occur (Nitao,1988): In the far field unsaturated zone, Weeks (1987) has described natural convection that occurs 
through Yucca Mountain due to seasonal temperature changes. Heat and mass transfer are also important in matching saturated zone models to temperature logs and pressure tests and in modeling enhanced convection from repository heating.

The transport processes at Yucca Mountain are very complex. Various adsorption mechanisms ranging from simple linear relations to nonlinear isotherms must be incorporated in the transport models. Multiple interacting chemical species must be modeled so that this structure can represent radioactive decay with daughter products and coupled geochemical transport.

The primary use of the FEHMN application will be to assist in the understanding of flow fields and mass transport in the saturated and unsaturated zones below the potential Yucca Mountain repository. Studies in the saturated zone are prescribed in YMP-LANLSP-8.3.1.2.3.1.7 (the-G-Wells project) and include use of the FEHMN code to design and analyze tracer tests (reactive-and-non-reactive) to characterize the flow field below Yucca Mountain. Studies in the unsaturated zone are prescribed in YMP-LANL-SP8.3.1.3.7.1 and include the study of coupled processes, (multicomponent flow-and-natural convection).

\subsection{STRUCTURE OF THE SYSTEM MODEL}

The sub-models that make up the overall transport model are:

Flow and Energy Transport Equations for simulation of processes within porous and permeable media which include:

- Heat conduction only;

- Heat and mass transfer with pressure and temperature dependent properties, relative permeabilities and capillary pressures;

- Isothermal air-water transport; and

- Heat and mass transfer with noncondensible gas.

Dual Porosity and Double Porosity / Double Permeability Formulation for problems dominated by fracture flow;

Solute Transport Models, including:

- A reactive transport model that simulates transport of multiple solutes with chemical reaction and

- A particle tracking model.

Constitutive Relationships for pressure and temperature dependent fluid/air/gas properties, relative permeabilities and capillary pressures, stress dependencies, and reactive and sorbing solutes which encompass:

- Thermodynamic equations,

- Air and Air/Water Vapor Mixtures,

- Equation of State Models,

- Relative Permeability and Capillary Pressure Functions, and

- Stress Dependent Properties.

\subsection{GENERAL NUMERICAL PROCEDURE}

The numerical solution strategy for FEHMN is shown in Figure 1. 


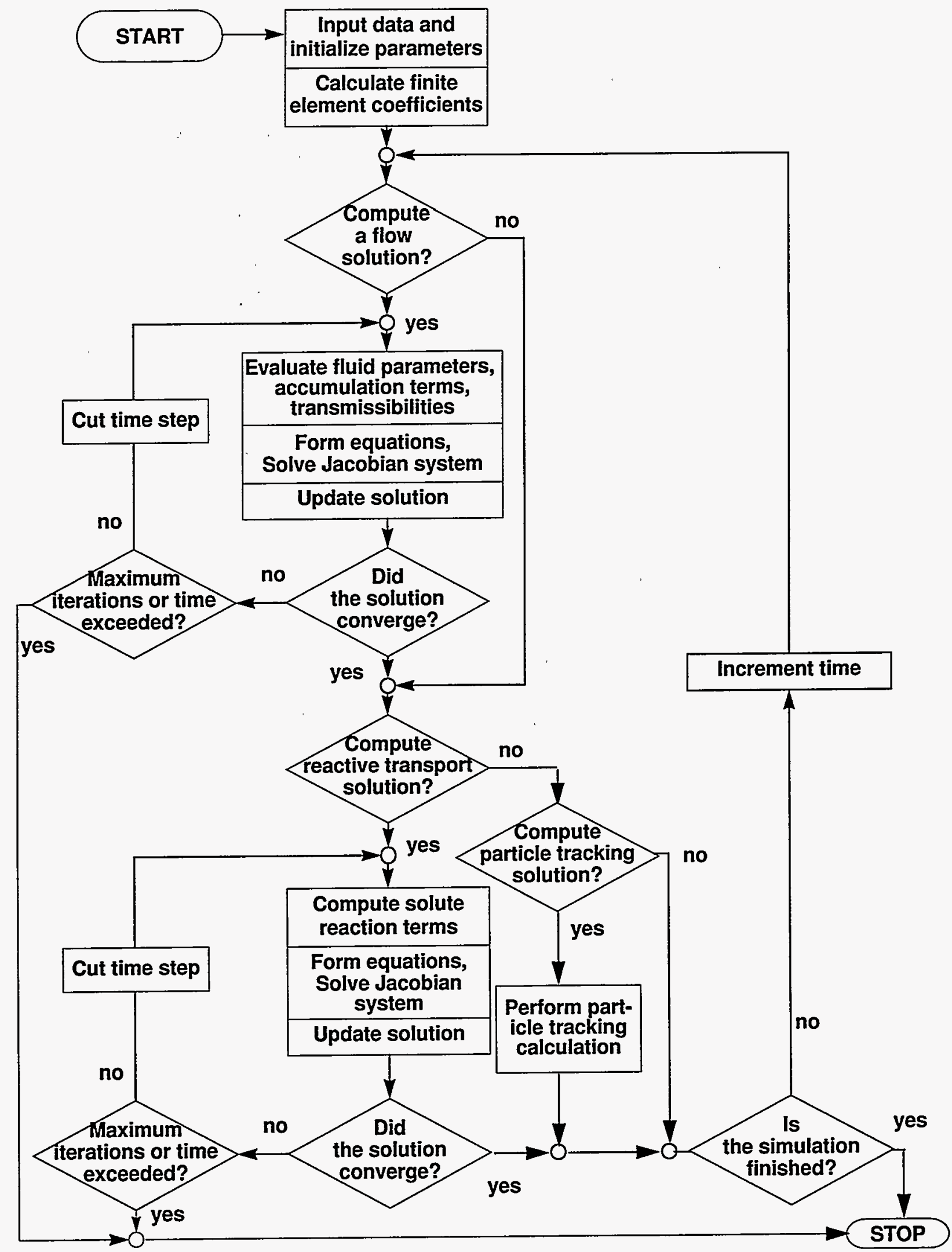

Figure 1. Simplified diagram of flow in the FEHMN application. 


\subsection{COMPONENT MODELS}

\subsection{Flow and Energy Transport}

\subsubsection{Purpose}

The purpose of this model is to simulate heat conduction, heat and mass transfer for multiphase flow within porous and permeable media, and noncondensible gas flow within porous and permeable media.

For heat conduction the input to the model consists of an initial description of the media (rock) properties and state. The output consists of a final media state.

For heat and mass transfer the input to the model consists of an initial description of the fluid state as well as media properties. The output consists of a final fluid state.

For noncondensible gas flow, in addition to the initial media properties and fluid state, the description of the initial state of gas is required. The output consists of the final state of gas in addition to that described for the previous components.

\subsubsection{Assumptions and Limitations}

The major assumptions are those associated with Darcy's law for fluid flow. This restricts the velocity of fluid flow to be very slow. The exact quantification of the values is best addressed in the associated validation report. Another main assumption is one of thermal equilibrium between the fluid and the rock (locally). This is usually an excellent assumption as the thermal wave for rocks travels on the order of $10^{-3} \mathrm{~m} / \mathrm{s}, 10^{-3} \mathrm{~m}$ is the upper limit of the pore size and fluid velocities are of the order of $10^{-5} \mathrm{~m} / \mathrm{s}$. Other assumptions include an immovable rock phase and negligible viscous heating. The assumptions associated with flow are discussed in Brownell (1975).

\subsubsection{Derivation}

Because the derivation of the governing equations is analogous for heat conduction, heat and mass transfer for multiphase flow within porous and permeable media, noncondensible gas flow within porous and permeable media, and transport of multiple solutes within porous and permeable media, only the heat and mass derivation will be presented.

Detailed derivations of the governing equations for two-phase flow including heat transfer have been presented by several investigators (e.g., Mercer and Faust, 1975, and Brownell, et al. 1975), and therefore only a brief development will be presented here. The notation used is given in Table I.

Conservation of mass for water is expressed by the equation

$$
\frac{\partial A_{m}}{\partial t}+\bar{\nabla} \cdot \overline{f_{m}}+q_{m}=0
$$

where the mass per unit volume, $A_{m}$, is given by 


$$
A_{m}=\phi\left(S_{v} \rho_{v}\left(1-\eta_{v}\right)+S_{l} \rho_{l}\left(1-\eta_{l}\right)\right)
$$

and the mass flux, $\overline{f_{m}}$, is given by

$$
\overline{f_{m}}=\left(1-\eta_{v}\right) \rho_{v} \overline{v_{v}}+\left(1-\eta_{l}\right) \rho_{l} \overline{v_{l}}
$$

Here $\phi$ is the porosity of the matrix, $S$ is saturation, $\rho$ is density, $\eta$ is the concentration of the noncondensible gas and is expressed as a fraction of the total mass, and $\bar{v}$ is velocity with the subscripts $v$ and $l$ indicating quantities for the vapor phase and the liquid phase, respectively. Source and sink terms (such as bores, reinjection wells, or groundwater recharge) are represented by the term $q_{m}$.

Conservation of fluid-rock energy is expressed by the equation

$$
\frac{\partial A_{e}}{\partial t}+\bar{\nabla} \cdot \bar{f}_{e}+q_{e}=0
$$

where the energy per unit volume, $A_{e}$, is given by

$$
A_{e}=(1-\phi) \rho_{r} u_{r}+\phi\left(S_{v} \rho_{v} u_{v}+S_{l} \rho_{l} u_{l}\right)
$$

with $u_{r}=c_{p r} T$, and the energy flux, $\bar{f}_{e}$, is given by

$$
\bar{f}_{e}=\rho_{v} h_{v} \overline{v_{v}}+\rho_{l} h_{l} \bar{v}_{l}-K \bar{\nabla} T
$$

Here the subscript $r$ refers to the rock matrix; $u_{r}, u_{v}$, and $u_{l}$ are specific internal energies; $c_{p r}$ is the specific heat; $h_{v}$ and $h_{l}$ are specific enthalpies; $K$ is an effective thermal conductivity; $T$ is the temperature; and $q_{e}$ is the energy contributed from sources and sinks.

To complete the governing equations it is assumed that Darcy's Law applies to the movement of each phase:

$$
\bar{v}_{v}=\frac{k R_{v}}{\mu_{v}}\left(\bar{\nabla} P_{v}-\rho_{v} \bar{g}\right)
$$

and

$$
\bar{v}_{l}=\frac{k R_{l}}{\mu_{l}}\left(\bar{\nabla} P_{l}-\rho_{l} \bar{g}\right) .
$$


Here $k$ is the permeability, $R_{v}$ and $R_{l}$ are the relative permeabilities, $\mu_{v}$ and $\mu_{l}$ are viscosities, $P_{v}$ and $P_{l}$ the phase pressures, and $g$ represents the acceleration due to gravity (the phase pressures are related by $P_{v}=P_{l}+P_{c a p}$, where $P_{c a p}$ is the capillary pressure). For simplicity, the equations are shown for an isotropic medium, though this restriction does not exist in the computer code.

Using Darcy's Law the basic conservation Equations (1) through (4) can be combined

$$
\begin{gathered}
-\bar{\nabla} \cdot\left(\left(1-\eta_{v}\right) D_{m v} \bar{\nabla} P_{v}\right)-\bar{\nabla} \cdot\left(\left(1-\eta_{l}\right) D_{m l} \bar{\nabla} P_{l}\right)+q_{m}+ \\
\frac{\partial}{\partial z} g\left(\left(1-\eta_{v}\right) D_{m v} \rho_{v}+\left(1-\eta_{l}\right) D_{m l} \rho_{l}\right)+\frac{\partial A_{m}}{\partial t}=0
\end{gathered}
$$

and

$$
\begin{gathered}
-\bar{\nabla} \cdot\left(D_{e v} \bar{\nabla} P_{v}\right)-\bar{\nabla} \cdot\left(D_{e l} \bar{\nabla} P_{l}\right)-\bar{\nabla} \cdot(K \bar{\nabla} T)+q_{e}+ \\
\frac{\partial}{\partial z} g\left(D_{e v} \rho_{v}+D_{e l} \rho_{l}\right)+\frac{\partial A_{e}}{\partial t}=0
\end{gathered}
$$

where $z$ is oriented in the direction of gravity. Here the transmissibilities are given by

$$
D_{m v}=\frac{k R_{v} \rho_{v}}{\mu_{v}}, D_{m l}=\frac{k R_{l} \rho_{l}}{\mu_{l}}
$$

and

$$
D_{e v}=h_{v} D_{m v}, D_{e l}=h_{l} D_{m l} .
$$

The source and sink terms in Equations (1) and (4) arise from bores, and if the total mass withdrawal, $q_{m}$, for each bore is specified, then the energy withdrawal, $q_{e}$, is determined as follows:

$$
q_{e}=q_{v} h_{v}+q_{l} h_{l}
$$

where

$$
q_{v}=v q_{m}, q_{l}=(1-v) q_{m}
$$

and 


$$
v=\frac{1}{1+\frac{\rho_{l} R_{l} \mu_{v}}{\rho_{v} R_{v} \mu_{l}}}
$$

The form of Equation (15) shows how important the relative permeability ratio $R_{l} / R_{v}$ is in controlling the discharge composition. Other source/sink terms arise from implementation of boundary conditions. These include specified pressure and temperatures and are discussed in Section 8.1.6, "Derivation of Numerical Model" subsection "Boundary Conditions". The relative permeability and capillary pressure functions are summarized in Section 8.4, "Constitutive Relationships".

The final form of the pure heat conduction equation is easily obtained from Equation (10) when all convective terms are eliminated:

$$
-\bar{\nabla} \cdot(K \bar{\nabla} T)+q_{e}+\frac{\partial A_{e}}{\partial t}=0
$$

The mass flux, $\bar{f}_{\eta}$, source (or sink) strength, $q_{\eta}$, and accumulation term, $A_{\eta}$, are defined as follows for the noncondensible gas conservation equation:

$$
\begin{aligned}
& \overline{f_{\eta}}=\eta_{v} \rho_{v} \overline{v_{v}}+\eta_{l} \rho_{l} \bar{v}_{l}, \\
& q_{\eta}=\eta_{v} q_{v}+\eta_{l} q_{l}, \\
& A_{\eta}=\phi\left(\eta_{v} S_{v} \rho_{v}+\eta_{l} S_{l} \rho_{l}\right) .
\end{aligned}
$$

The noncondensible gas conservation equation is

$$
\begin{gathered}
-\bar{\nabla} \cdot\left(\eta_{v} D_{m v} \bar{\nabla} P_{v}\right)-\bar{\nabla} \cdot\left(\eta_{l} D_{m l} \bar{\nabla} P_{l}\right)-\bar{\nabla} \cdot\left(D_{v a} \bar{\nabla} \eta_{v}\right)+q_{\eta}+ \\
\frac{\partial}{\partial z} g\left(\eta_{v} D_{m v} \rho_{v}+\eta_{l} D_{m l} \rho_{l}\right)+\frac{\partial A_{\eta}}{\partial t}=0 .
\end{gathered}
$$

Here $\eta$ is the concentration of the noncondensible gas and is expressed as a fraction of the total mass. As with the water balance equations, source/sink terms are used to implement boundary conditions. The reader is referred to Section 8.1.6, "Derivation of Numerical Model" subsection "Boundary Conditions" for details. The air water diffusivity is given by

$$
D_{v a}=\tau \phi S_{v} D_{v a}^{0} \rho_{v} \frac{0.101325}{P}\left[\frac{T+273.15}{273.15}\right]^{\theta}
$$


where $\tau$ is the tortuosity factor and $D_{v a}^{0}$ is the value of $D_{v a}$ at standard conditions. Within FEHMN the value of $D_{v a}$ is set to $2.4^{*} 10^{-5} \mathrm{~m}^{2} / \mathrm{s}, \theta$ is set to 2.334 , and the tortuosity factor is an input parameter.

The Equations (9), (10), (16), and (20) represent the model equations for fluid and energy transport in the computer code FEHMN. It should be noted that Equation (9) also represents pure water with $\eta$ set to 0 .

For situations in which heat effects are minimal, the model can be simplified. The isothermal air-water two phase system in FEHM is represented somewhat differently then the nonisothermal system defined above. Here the liquid phase is pure water and the vapor phase is pure air. The component mass balance equations are then also phase balance equations:

$$
\begin{aligned}
& \frac{\partial}{\partial t}\left(\phi \rho_{l} S_{l}\right)-\bar{\nabla} \cdot\left(D_{m l} \bar{\nabla} P_{l}\right)+q_{m l}+\frac{\partial}{\partial z} g\left(D_{m l} \rho_{l}\right)=0 \\
& \frac{\partial}{\partial t}\left(\phi \rho_{v} S_{v}\right)-\bar{\nabla} \cdot\left(D_{m v} \bar{\nabla} P_{v}\right)+q_{m v}+\frac{\partial}{\partial z} g\left(D_{m v} \rho_{v}\right)=0 .
\end{aligned}
$$

where Equation (22) is the water balance equation and Equation (23) refers to the conservation of air. Here the subscript $l$ refers to the liquid water properties and $v$ refers to air properties. One option in the model is to solve Equations (22) and (23) as a full two-phase flow problem. A further simplification can be made in which the air pressure is assumed to be constant. This leads to an equation which is similar to the Richard's equation for unsaturated flow. The method reduces to using only Equation (22). The method is described further in Section 8.16 subsection "Reduced Degree of Freedom Algorithms".

\subsubsection{Applications}

The component model described above may be used to model the flow of air, water, water vapor, and heat in a porous medium. The validity of the model is dependent on the validity of the equations described in Section 8.1.3. The flow of both air and water must be sufficiently small at all possible flow rates so that the above described equations will be valid. This is believed to be the case at Yucca Mountain. Of more concern is the accuracy of the required input and the numerical precision to which these equations are able to be solved.

For the flow equations, the saturated permeabilities, porosities, and fracture permeabilities and volumes of hydrogeologic units are required. In addition, the relative permeability and capillary pressure functions are also required. Historically this information has been difficult to obtain. It is important to note that the capillary pressure at low liquid saturations is very important to the validity of the calculations but is not available in regions near the residual saturations. 
The issue of numerical accuracy is extremely important to the usefulness of the results. The accuracy may be evaluated by solving the same problem using different size grids and evaluating the change in the solution.

\subsubsection{Numerical Method Type}

The primary numerical method used in FEHMN is the Finite Element Method. The reader is referred to Zienkiewicz (1977) for an excellent account of the method. The summary of the numerics in FEHMN given in Section 8.1.6 assumes a basic knowledge of the numerical solution of Partial Differential Equations. In addition a working knowledge of the Finite Element method is helpful.

\subsubsection{Derivation of Numerical Model}

Discretization: The time derivatives in Equations (9), (10), (16), (20), and (76) are discretized using the standard first order method (Hinton and Owen, 1979) given by

$$
f\left(t^{n+1}\right)=f\left(t^{n}\right)+\Delta t\left[w f^{\prime \prime}\left(t^{n+1}\right)+(1-w) f^{\prime \prime}\left(t^{n}\right)\right]
$$

where $f\left(t^{n+1}\right)$ is the desired function at time $t^{n+1}, f\left(t^{n}\right)$ is the known value of $f$ at time $t^{n}, \Delta t$ is the time step, $f^{\prime \prime}$ is the derivative of $f$ with respect to time and $w$ is a weighting factor. For $w=1$, the scheme is fully implicit (backward Euler) and for $w=0$, the scheme is fully explicit (forward Euler).

The space derivatives in the governing equations are discretized using the finite element formulation. The finite element equations are generated using the Galerkin formulation. For a detailed presentation of the finite element method the reader is referred to Zienkiewicz (1977). In this method the flow domain, $\Omega$, is assumed to be divided into finite elements; and variables $P, T$, and $\eta$, along with the accumulation terms $A_{m}, A_{e}$, and $A_{\mathfrak{\eta}}$ are interpolated in each element: $P_{v}=[N]\left\{P_{v}\right\}, P_{l}=[N]\left\{P_{l}\right\}$, $T=[N]\{T\}, \eta_{v}=[N]\left\{\eta_{v}\right\}, A_{m}=[N]\left\{A_{m}\right\}, A_{e}=[N]\left\{A_{e}\right\}$, and $A_{\eta}=[N]\left\{A_{\eta}\right\}$ where $[N]$ is the shape function.

These approximations are introduced in Equations (9), (10), (16), and (20), and the Galerkin formulation (described by Zienkiewicz and Parekh, 1973) is applied. The following equations are derived: 


$$
\begin{aligned}
& {\left[T_{m, v}\right]\left\{P_{v}\right\}+\left[T_{m, l}\right]\left\{P_{l}\right\}+[\hat{C}]\left\{\frac{\partial A_{m}}{\partial t}\right\}+\left\{q_{m}\right\}-} \\
& g\left\{G_{m, v}\right\}-g\left\{G_{m, l}\right\}=\left\{F_{m}\right\}, \\
& {\left[T_{e, v}\right]\left\{P_{v}\right\}+\left[T_{e, l}\right]\left\{P_{l}\right\}+[K]\{T\}+[\hat{C}]\left\{\frac{\partial A_{e}}{\partial t}\right\}+} \\
& \left\{q_{e}\right\}-g\left\{G_{e, v}\right\}-g\left\{G_{e, l}\right\}=\left\{F_{e}\right\}
\end{aligned}
$$

and

$$
\begin{gathered}
{\left[T_{\eta, v}\right]\left\{P_{v}\right\}+\left[T_{\eta, l}\right]\left\{P_{l}\right\}+\left[D_{v a}\right]\left\{\eta_{v}\right\}+[\hat{C}]\left\{\frac{\partial A_{\eta}}{\partial t}\right\}+} \\
\left\{q_{\eta}\right\}-g\left\{G_{\eta, v}\right\}-g\left\{G_{\eta, l}\right\}=\left\{F_{\eta}\right\}
\end{gathered}
$$

where

$$
\begin{aligned}
& T_{m i j}=\int_{\Omega} \bar{\nabla} N_{i} \cdot D_{m}^{U P} \bar{\nabla} N_{j} d V, \\
& T_{e i j}=\int_{\Omega} \bar{\nabla} N_{i} \cdot D_{e}^{U P} \bar{\nabla} N_{j} d V, \\
& T_{\eta i j}=\int_{\Omega} \bar{\nabla} N_{i} \cdot D_{m} \eta{ }^{U P} \bar{\nabla} N_{j} d V, \\
& K_{i j}=\int_{\Omega} \bar{\nabla} N_{i} \cdot \hat{K} \bar{\nabla} N_{j} d V, \\
& \hat{C}_{i j}=\int_{\Omega} N_{i} N_{j} d V, \\
& G_{m i}=\int_{\Omega} \frac{\partial N_{i}}{\partial z} N_{j} D_{m}^{U P} \rho_{m} d V,
\end{aligned}
$$




$$
G_{e i}=\int_{\Omega}^{\partial N_{i}} \frac{N_{j}}{\partial z} N_{e}^{U P} \rho_{e} d V
$$

and

$$
G_{\eta i}=\int_{\Omega}^{\partial N_{i}} \frac{\partial}{\partial z} N_{j} D_{m} \eta^{U P} \rho_{e} d V
$$

In the above equations $\hat{K}=\frac{2 K_{i} K_{j}}{K_{i}+K_{j}}$, and the $D^{U P}$ terms indicate an upstream-weighted transmissibility (Dalen, 1979). This technique has worked well in the low-order elements (3-node triangle, 4-node quadrilateral), where the schemes resemble difference techniques. The upstream weighting is determined by evaluating the internode flux for the nodes $i$ and $j$. The shape function coefficients are generated in a unique way that requires the integrations in Equations (33), (34), and (35) to be performed only once and the nonlinear coefficients to be separated from this integration. The reader is referred to Zyvoloski (1983) for more details.

The integration schemes available in FEHMN are Gauss integration and a node point scheme used by Young (1981). His implementation differs from common methods in that it uses Lobatto instead of Gauss integration. The net effect is that, while retaining the same order of integration accuracy (at least for linear and quadratic elements), there are considerably fewer nonzero terms in the resulting matrix equations. Figure 2 shows a comparison of the nodal connections for Lobatto and Gauss integration methods. It should be noted that these results hold on an orthogonal grid only. If a nonorthogonal grid were introduced, then additional nonzero terms would appear in the Lobatto quadrature method. Note also that the linear elements yield the standard 5- or 7-point difference scheme. The reader is referred to Young's paper for more details.

In addition to the Finite Element integration techniques described above, the code has provisions for Finite Volume calculation of the internode flow terms described by Equations (28) - (35). In the Finite Volume approach, the geometric terms are calculated as area projections and distances between nodes. The geometric part of Equations (28), (29), and (30) are given by the area between the nodes divided by the distance. The area is partitioned according to the perpendicular bisectors of the midpoints of the sides of the elements. This is shown in Fig. 3 for triangles in two dimensions. An analogous approach is used in three dimensions for tetrahedrals. Quadrilaterals in two dimensions and hexahedrals in three dimensions are first decomposed into triangles and tetrahedrals, respectively, and the geometry coefficients formed as described above. For more details the reader is referred to Fung, et al. (1994).

It is important to note here that with upwinding, the geometric factors that govern internode flow, regardless of whether calculated from a Finite Element or Finite Volume approach, must not change in sign. This requires a Delaunay grid. For two dimensions, this requires the angles in the 


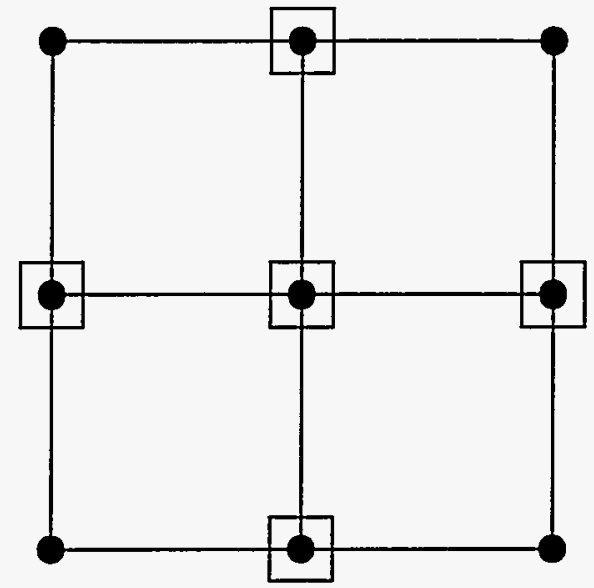

Figure 2. Comparison of nodal connections for conventional ( $\bullet$ ) and Lobatto $(\square)$ integrations for an orthogonal grid.

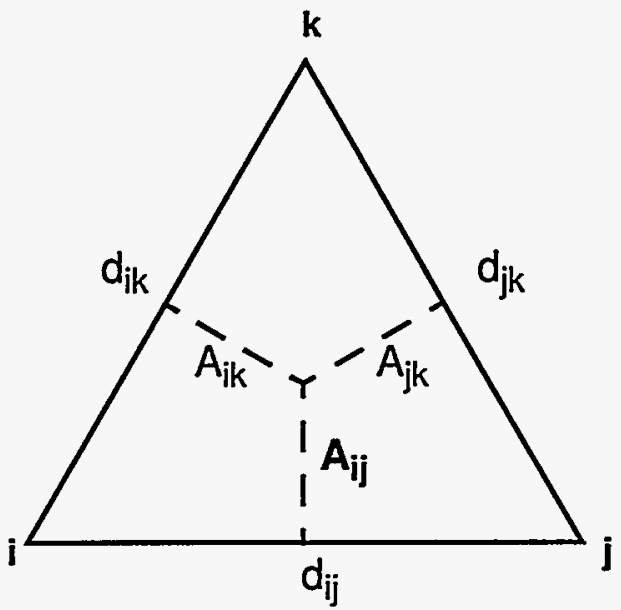

Figure 3. Area projections and internode distances used in Finite Volume calculations on a Delaunay grid.

triangles to be less than $\pi / 2$ radians for a triangle on the boundary and $\pi$ radians for an interior triangle. The reader is again referred to Fung, et al. (1994) for more details.

The development of the numerical approximation of the transport equation is similar to that for the flow equations. Following the discussion above, the species concentration, $C$, and the species accumulation term, $A_{c}$, are interpolated in each element: $C=[N]^{T}\{C\}, A_{c}=[N]^{T}\left\{A_{c}\right\}$.

Using these approximations and a Galerkin approach, the following equation is obtained 


$$
\begin{gathered}
{\left[T_{c}(C)\right]\{P\}+\left[D_{c}\right]\{C\}+[\hat{C}]\left\{\frac{\partial A_{c}}{\partial t}\right\}+\left\{q_{c}\right\}+} \\
g\left\{G_{c}\right\}=\left\{F_{c}\right\}
\end{gathered}
$$

where

$$
\begin{aligned}
& T_{c i j}=\int_{\Omega} \bar{\nabla} N_{i} \cdot D_{m} C_{i j}^{U P} \bar{\nabla} N_{j} d V, \\
& D_{c i j}=\int_{\Omega} \bar{\nabla} N_{i} \cdot D_{c} \bar{\nabla} N_{j} d V, \\
& G_{c i j}=\int_{\Omega} \frac{\partial N_{i}}{\partial z} \cdot D_{m} C^{U P} \bar{\nabla} N_{j} d V,
\end{aligned}
$$

and $D_{m} C^{U P}$ is an upstream weighted concentration transmissibility. This approach is similar to the finite difference method for solving the transport equations.

Boundary Conditions: Two types of fluid (mass) sources and sinks are implemented: a specified-flow-rate source/sink and a specified-pressure condition at a source/sink. No-flow or impermeable boundary conditions are automatically satisfied by the finite element mesh. The constant pressure boundary condition is implemented using a pressure dependent flow term

$$
q_{m, i}=I_{m, i}\left(P_{\text {flow }, i}-P_{i}\right)
$$

where $P_{i}$ is the pressure at the source node $i, P_{\text {flow, } i}$ is the specified flowing pressure, $I_{m, i}$ is the impedance, and $q_{m, i}$ is the mass flow rate. By specifying a large $I$ the pressure can be forced to be equal to $P_{\text {flow }}$. The energy (temperature) specified at a source/sink or flowing pressure node refers only to the incoming fluid value, if fluid flows out, stability dictates that the energy of the in-place fluid be used in calculations.

In addition to the mass flow source/sink, heat flow sources can also be provided. A specified heat flow can be input or a specified temperature obtained

$$
q_{e, i}=I_{e, i}\left(T_{\text {flow, } i}-T_{i}\right)
$$

where $T_{i}$ is the temperature at the source node $i, T_{\text {flow, } i}$ is the specified flowing temperature, $I_{e, i}$ is the impedance to heat flow (thermal resistance), and $q_{e, i}$ is the heat flow. This heat flow is superimposed on any 
existing heat flow from other boundary conditions or source terms. Specified saturations, relative humidities, air mass fractions as well as specified air flows are allowed. These use source/sinks to achieve the desired variable values in a way analogous to that described for pressure boundary conditions.

In FEHMN there is also a provision for creating large volume reservoirs which effectively hold variables at their initial values. The nodes are labeled on input and the volumes replaced after the calculation of the geometric coefficients with a reservoir volume of $10^{13} \mathrm{~m}^{3}$.

Solution Method: The application of the discretization methods to the governing partial differential equations yields a system of nonlinear algebraic equations. To solve these equations, the Newton-Raphson iterative procedure is used. This is an iterative procedure that makes use of the derivative information to obtain an updated solution from an initial guess. Let the set equations to be solved be given by

$$
\{F\}(\{x\})=\{0\}
$$

where $\{x\}$ is the vector of unknown values of the variables that satisfy the above equation. The procedure is started by making an initial guess at the solution, say $\{x\}^{0}$. This is usually taken as the solution from the previous time step. Denoting the value of $\{x\}$ at the $k t h$ iteration by $\{x\}^{k}$, the updating procedure is given by

$$
\{x\}^{k+1}=\{x\}^{k}-\{F\}^{k}\left(\frac{\partial\{F\}}{\partial\{x\}^{k}}\right)^{-1} .
$$

At each step, the residuals $\{F\}^{k}=\{F\}\left(\{x\}^{k}\right)$ are compared with a prescribed error tolerance. The prescribed error tolerance, $\varepsilon$, is an input parameter and an $l^{2}$ norm is used:

$$
\|F\|_{k}=\left(\sum_{i} F_{i}^{2}\right)_{k}^{1 / 2}
$$

Convergence is achieved when

$$
\|F\|_{k} \leq \varepsilon\|F\|_{o} \text {. }
$$

$\varepsilon$ is usually in the range $10^{-4}-10^{-7}$. Semiautomatic timestep control is designed based on the convergence of the Newton iterations. If the code is unable to find a solution $\{x\}^{k}$ such that the residuals become less than the tolerance within a given number of iterations, the time step is reduced and the procedure repeated. On the other hand, if convergence is rapid, the 
timestep is increased by multiplying with a user supplied factor, thus allowing for large timesteps when possible.

The linear equation set to be solved at each Newton-Raphson iteration of Equation (43) is

$$
\left(\frac{\partial\{F\}}{\partial\{x\}^{k}}\right)\{\Delta x\}^{k+1}=-\{F\}^{k}
$$

where $\left(\frac{\partial\{F\}}{\partial\{x\}^{k}}\right)$ is the Jacobian matrix, $\{\Delta x\}^{k+1}$ is the change in the solution vector $\left\{\Delta x^{k+1}=x^{k+1}-x^{k}\right\}$, and $\{F\}^{k}$ is the residual. It is solved with a reuse component, GZSOLVE, that provides a robust solution method for sparse systems of equations. Further details of the solution procedure can be found in the GZSOLVE MMS.

Reduced Degree of Freedom Algorithms: In the coupled physical processes that describe flow in porous media, often one process is dominant. In heat and fluid flow, for example, the pressure changes more rapidly than the temperature. This fact may be used to simplify the linear equations solved at each step of a Newton-Raphson iteration and was recognized by Zyvoloski, et al. (1979). Solving the pure water heat and mass flow leads to the following set of linear equations at each NewtonRaphson iteration:

$$
\left[\begin{array}{cc}
A_{m P} & A_{m T} \\
A_{e P} & A_{e T}
\end{array}\right]\left\{\begin{array}{c}
\Delta P \\
\Delta T
\end{array}\right\}=-\left\{\begin{array}{c}
F_{m} \\
F_{e}
\end{array}\right\}
$$

The subscripts $m$ and $e$ refer to the mass and energy balance equations respectively. The subscripts $P$ and $T$ refer to derivatives with respect to pressure and temperature respectively. The superscripts indicating iteration number have been dropped for convenience. From Equations (9) and (10) it can be seen that the primary contribution of temperature is to affect the thermal conduction terms and the density and viscosities. Pressure, however, affects the density and is directly involved in the Darcy velocities. In other words, the pressure more directly affects the global transport of heat and mass. Guided by this reasoning, a computationally efficient scheme is obtained by neglecting the off-diagonal derivatives with respect to temperature. With this modification the temperature change may be solved for using

$$
\{\Delta T\}=\left[A_{e T}\right]^{-1}\left\{-\left\{F_{e}\right\}-\left[A_{e P}\right]\{\Delta P\}\right\}
$$


This may in turn be substituted in the mass balance portion of Equation (47) giving

$$
\begin{gathered}
{\left[\left[A_{m P}\right]-\left[A_{m T}\right]\left[A_{e T}\right]^{-1}\left[A_{e P}\right]\right]\{\Delta P\}=} \\
-\left\{F_{m}\right\}+\left[A_{m T}\right]\left[A_{e T}\right]^{-1}\left\{F_{e}\right\} .
\end{gathered}
$$

The indicated matrix inversions and multiplications are performed with diagonal matrices and the resulting matrix for the calculation of the pressure correction is a banded matrix of exactly the same structure as $\left[A_{m P}\right]$. It was found that additional efficiency could be achieved by taking several passes of SOR iterations after the system in Equations (48) and (49) were solved (Bullivant and Zyvoloski,1990).

The same process can be used to reduce the air/water/heat coupled system to a one or two degree of freedom problem. Here the coupled $3 n$ by $3 n$ system may be written as

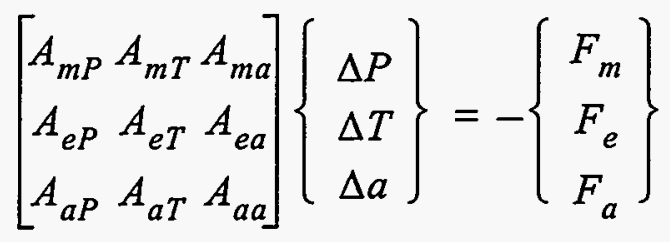

Here the subscript $a$ refers to the conservation of air mass and derivatives with respect to the air variable. The air variable is eliminated in favor of the pressure and temperature using

$$
\{\Delta a\}=\left[A_{a a}\right]^{-1}\left\{-\left\{F_{a}\right\}-\left[A_{a P}\right]\{\Delta P\}-\left[A_{e T}\right]\{\Delta T\}\right\} .
$$

Substituting this in the mass and energy correction equations:

$$
\begin{gathered}
\left.\left[\begin{array}{l}
\left.\left[A_{m P}\right]-\left[A_{m a}\right]\left[A_{a a}\right]^{-1}\left[A_{a P}\right]\right]\left[\left[A_{m T}\right]-\left[A_{m a}\right]\left[A_{a a}\right]^{-1}\left[A_{a T}\right]\right] \\
{\left[\left[A_{e P}\right]-\left[A_{e a}\right]\left[A_{a a}\right]^{-1}\left[A_{a P}\right]\right]\left[\left[A_{e T}\right]-\left[A_{e a}\right]\left[A_{a a}\right]^{-1}\left[A_{a T}\right]\right]}
\end{array}\right] \begin{array}{c}
\Delta P \\
\Delta T
\end{array}\right\} \\
=\left\{\begin{array}{l}
-\left\{F_{m}\right\}+\left[A_{m a}\right]\left[A_{a a}\right]^{-1}\left\{F_{a}\right\} \\
-\left\{F_{e}\right\}+\left[A_{e a}\right]\left[A_{a a}\right]^{-1}\left\{F_{a}\right\}
\end{array}\right\}
\end{gathered}
$$


During the simulation, the phase state of the system can change. This makes it necessary to rearrange Equations (51) and (52). The method remains the same. The reduced Equations (51) and (52) are useful in thermal simulations where phase changes or other factors reduce the timestep. The $3 n$ by $3 n$ system may further be reduced to an $n$ by $n$ system. This is discussed in Bullivant and Zyvoloski (1991). Bullivant and Zyvoloski also showed that the operations given above can conveniently be done during the equation normalization process.

The last reduced degree of freedom algorithm to be described reduces the isothermal air-water problem to a one variable system. The result is similar to the Richard's solution. To obtain a computationally efficient scheme, the air pressure is constrained to atmospheric pressure in the twophase region and the liquid saturation is constrained to 1.0 in the onephase liquid region. The method involves switching variables and associated derivatives in the solution of the linear system that produces the Newton-Raphson correction. The matrix equation that describes the Jacobian matrices for an isothermal system is given by

$$
\left[A_{w P}\right]\{\Delta P\}+\left[A_{w S}\right]\{\Delta S\}=-\left\{F_{w}\right\}
$$

Here the subscript $w$ refers to the water conservation equation and the subscripts $P$ and $S$ refer to derivatives with respect to pressure and saturation, respectively. Though Equation (53) has the appearance of being under constrained, for every matrix position there is only one non-zero entry in the two matrices $\left[A_{w P}\right]$ and $\left[A_{w S}\right]$. This is a consequence of the variable switching just discussed. The algorithm consists of replacing terms in $\left[A_{w P}\right]$ with terms from $\left[A_{w S}\right]$ if two-phase conditions exist at a node. The resulting system is

$$
\left[A_{w x}\right]\{x\}=-\left\{F_{w}\right\}
$$

where $x$ represents pressure or saturation depending on the nodal phase state.

\subsubsection{Location}

The implementation sequence for the Flow and Energy Transport Equations may be seen in Fig. 1. The box 'Form Equations, Solve Jacobian System' indicates the position in the algorithm of the components of the Flow equations in the overall structure of FEHMN.

\subsubsection{Numerical Stability and Accuracy}

The equations which are solved are highly nonlinear and coupled. The stability of the system has been maximized by solving the fully coupled and fully implicit formulation of the problem. Because of the nonlinearity, however, stability cannot be guaranteed. Logic has been incorporated to 
restart a timestep if the code realizes it is calculating in an area where the equation of state (as implemented by FEHMN) is not valid

Accuracy of the simulations is also clouded by the nonlinearity issue. Formally the spatial differencing is second order accurate and the time terms are first order accurate. There is a provision (which is usually invoked) which upwinds the transmissibility terms. This reduces the spatial accuracy to first order. It is difficult in practice to estimate the quality of a simulation from these theoretical considerations. The user is advised to run a given problem with several grid sizes and time step sizes to assess the quality of a particular solution obtained with FEHMN. The accuracy of the calculations is also addressed in the FEHMN verification report (Zyvoloski and Dash, 1991).

\subsubsection{Alternatives}

The primary alternative to the formulation given here is an integrated finite difference formulation. The reader is referred to Nitao, et al. (1988) and Pruess (1987) for details. The basic difference in theory is that FEHMN uses a node centered approach whereas the integrated finite difference formulation uses a cell centered approach. Classical finite differences may also be used to solve the equations presented herein but lack the geometric flexibility of the methods mentioned.

\subsection{Dual Porosity and Double Porosity / Double Permeability Formulation}

\subsubsection{Purpose}

Many problems are dominated by fracture flow. In these cases the fracture permeability controls the pressure communication in the reservoir even though local storage around the fracture may be dominated by the porous rock which communicates only with the closest fractures. This phenomena requires a model in which the fractures dominate the global pressure response of the reservoir. The fractures are needed merely as storage. Moench (1984) has studied several wells in the saturated zone beneath Yucca Mountain and found that results could be understood if dual porosity methods were used. The numerical model in which the matrix material is constrained to communicate only in the neighboring fractures is known as the Dual Porosity method.

In a partially saturated porous medium, flow is often dominated by capillary suction. In a medium comprised of fractures and matrix, the matrix material has the highest capillary suction and under relatively static conditions the moisture resides in the matrix material. Infiltration events, such as severe rainfall, can saturate the porous medium allowing rapid flow in the fractures. To capture this flow phenomena, a system of equations allowing communication between the fractures and matrix blocks in the reservoir in addition to the flow within the fractures and matrix blocks is necessary. This method is known as the Double Porosity / Double Permeability method.

The decision about which fracture model to use is often affected by the transient nature of the simulation. It is possible to obtain nearly the same results for a double permeability simulation using a less expensive equivalent continuum approach for a steady state solution but different results would be obtained for a transient solution. 
For transport, the alternative fracture formulations are even more important. Here the simulations are almost always transient. The matrix and fractures are in approximate pressure equilibrium and there is little flow from matrix to fracture. The tracer in this scenario is constrained to stay in the fracture if it started there. This often produces erroneous results that can be improved if diffusion from matrix to fracture is included. The fracture formulations in FEHMN account for matrix to fraction diffusion.

\subsubsection{Assumptions and Limitations}

In the Dual Porosity method, the computational volume consists of a fracture which communicates with fractures in other computational cells, and matrix material which only communicates with the fracture in its own computational cell. This behavior of the matrix material is both a physical limitation and a computational tool. The physical limitation results from the model's inability to allow the matrix materials in different cells to communicate directly. This yields only minor errors in saturated zone calculations, but could pose larger errors in the unsaturated zone where capillary pressures would force significant flow to occur in the matrix material. The computational advantages will be addressed in Section 8.2.3.

The Double Porosity / Double Permeability method differs from the Dual Porosity method in that the matrix can communicate with other matrix nodes. This produces a more realistic simulation but is computationally more expensive.

\subsubsection{Derivation}

Figure 4 depicts the double porosity / double permeability and dual porosity concepts. Two parameters characterize a double porosity / double permeability reservoir. The first is the volume fraction, $V_{f}$, of the fractures in the computational cell. For the single matrix node system shown in

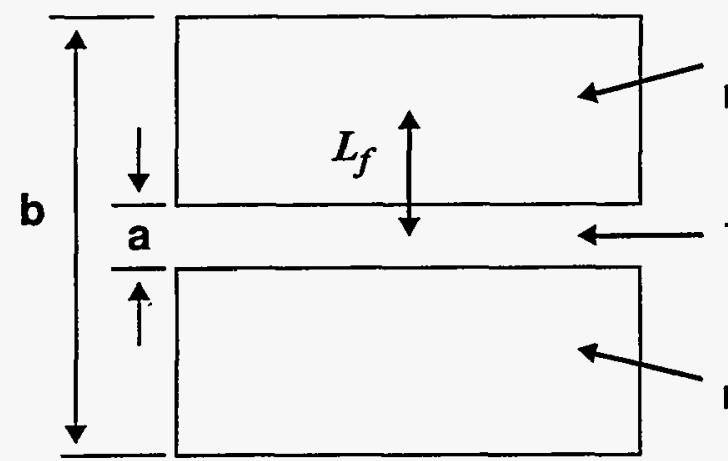

One matrix node (double porosity / double permeability)

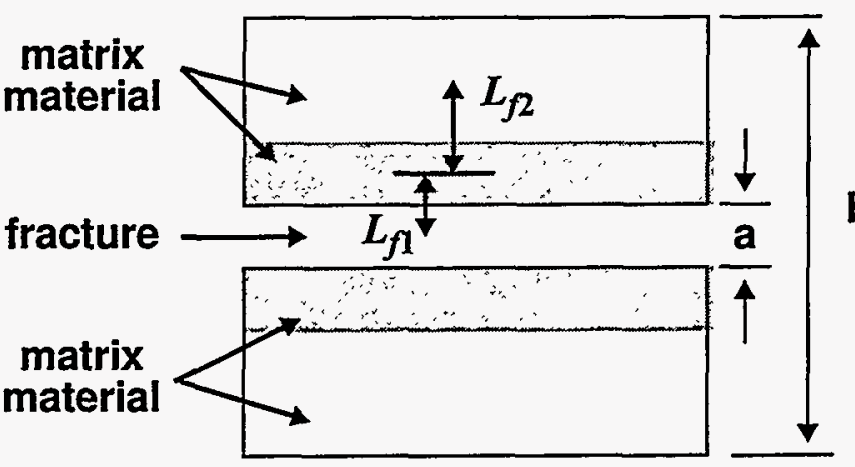

Two matrix nodes (dual porosity)

Figure 4. Computational volume elements showing dual porosity and double porosity / double permeability parameters. 
Fig. 4 this fraction is $a / b$. The second parameter is related to the fracture's ability to communicate with the local matrix material. In the literature this parameter takes a variety of forms. The simplest is a length scale, $L_{f}$ This quantifies the average distance the matrix material is from the fracture. With just one node in the matrix material the transient behavior in the matrix material cannot be modeled. To improve this situation, two nodes are used in FEHMN to represent the matrix material for a dual porosity reservoir. Conceptually, this is the same formulation as just described with the addition of a second fracture volume (it is assumed the length scale of each matrix volume is proportional to the volume fraction). This is the two matrix node system shown in Fig. 4. More matrix nodes could be added, but data is rarely good enough to justify the use of even two matrix nodes. The simple slab model depicted in Fig. 4 is just one of several different geometric arrangements. Moench (1984) and Warren and Root (1962) list other reservoir types. All of them are similar in the assumption of a local one dimensional connection of the matrix to the fracture.

A volume fraction and length scale are used to characterize the system. Equations (9), (10), (20), and (76) are formulated for both the fracture and matrix computational grids. One dimensional versions are created to locally couple the two sets of equations. The length scales are used to modify spatial difference terms and the volume fractions are used to modify the accumulation terms.

The volume fractions for the double porosity / double permeability formulation satisfy the following relationship

$$
V_{f}+V_{f}=1
$$

where $V_{f}$ is the volume fraction of fractures and $V_{f 1}$ is the fraction of the matrix volume. The length scales are partitioned for the fracture and matrix volumes using

$$
\begin{aligned}
& L_{f}=L_{f 0} V_{f} \\
& L_{f 1}=L_{f 0} V_{f 1}
\end{aligned}
$$

where $L_{f}$ is the length scale for the fracture volume, $L_{f 1}$ is the length scale of the matrix volume, and $L_{f 0}$ is a characteristic length scale.

The volume fractions for the dual porosity formulation satisfy the following relationship

$$
V_{f}+V_{f 1}+V_{f 2}=1
$$

where $V_{f}$ is the volume fraction of fractures, $V_{f 1}$ is the fraction of the first matrix volume, and $V_{f 2}$ is the fraction of the second matrix volume. Recall that two nodes are used to model the porous rock (matrix) and the matrix material communicates only with the local fractures. The length scales are given by 


$$
\begin{aligned}
& L_{f}=L_{f 0} V_{f} \\
& L_{f 1}=L_{f 0} V_{f 1} \\
& L_{f 2}=L_{f 0} V_{f 2}
\end{aligned}
$$

where $L_{f}$ is the length scale for the fracture volume, $L_{f 1}$ is the length scale of the first matrix volume, $L_{f 2}$ is the length scale of the second matrix volume, and $L_{f 0}$ is a characteristic length scale.

\subsubsection{Application}

The fracture models are extremely useful in investigating flow and transport in the geologic repository because of the importance of fracture flow and transport. Large differences are expected between transport calculations from lumped (matrix and fracture) properties models and models that include fracture flow and transport. FEHMN through a realistic description of fractures, allows the use of more realistic radionuclide dose calculations in the performance assessment calculations.

\subsubsection{Numerical Method Type}

Only algebraic manipulations are used in the derivations described in Section 8.2.6.

\subsubsection{Derivation of Numerical Model}

\subsubsection{Dual porosity}

Computationally, the volume fractions and length scales are used to create one dimensional versions of Equations (9), (10), (20), and (76). The length scale is used to modify spatial difference terms and the volume factors are used to modify the accumulation terms [the $\hat{C}$ matrix in Equations (25) and (26)].

The geometric factor representing the spatial differencing of the one dimensional equation for flow between the fracture and the first matrix node [analogous to the geometric part of Equations (28) and (29)] is given by

$$
T_{f f 1}=\frac{V_{T}}{L_{f 1}\left(L_{f}+L_{f 1}\right)}
$$

where $V_{T}$ is the total volume of the computational cell.

The analogous term for the flow from the first matrix volume to the second matrix volume is given by

$$
T_{f 1 f 2}=\frac{V_{T}}{L_{f 2}\left(L_{f 1}+L_{f 2}\right)}
$$


Using these geometric factors Equations (25), (26), and (27) are modified with the addition of the following flux terms

$$
\begin{aligned}
& T_{f 1 f 2}\left(\frac{k \rho_{v}}{\mu_{v}}\left(P_{m, v}-P_{f, v}\right)+\frac{k \rho_{l}}{\mu_{l}}\left(P_{m, l}-P_{f, l}\right)\right), \\
& T_{f 1 f 2}\left(\frac{k \rho_{v} h_{v}}{\mu_{v}}\left(P_{m, v}-P_{f, v}\right)+\frac{k \rho_{l} h_{l}}{\mu_{l}}\left(P_{m, l}-P_{f, l}\right)\right),
\end{aligned}
$$

and

$$
T_{f 1 f 2}\left(\frac{k \rho_{v} h_{v} \eta_{v}}{\mu_{v}}\left(P_{m, v}-P_{f, v}\right)+\frac{k \rho_{l} h_{l} \eta_{l}}{\mu_{l}}\left(P_{m, l}-P_{f, l}\right)\right)
$$

where $m$ refers to the matrix and $f$ to the fracture. The equation for the matrix consists of these transfer terms plus the accumulation terms analogous to those for the fracture and shown in Equations (2), (5), (19), and (24). It should also be noted that the gravity terms are not shown in the transfer terms above for simplicity but are represented in an analogous way.

The one dimensional nature of the equations provides a computationally efficient method to solve the algebraic equations arising from the dual porosity simulation. Equation (64) shows the matrix equation arising from a dual porosity simulation.

$$
\left[\begin{array}{lll}
A_{00} & A_{01} & A_{02} \\
A_{10} & A_{11} & A_{12} \\
A_{20} & A_{21} & A_{22}
\end{array}\right]\left\{\begin{array}{l}
x_{0} \\
x_{1} \\
x_{2}
\end{array}\right\}=-\left\{\begin{array}{l}
b_{0} \\
b_{1} \\
b_{2}
\end{array}\right\}
$$

Here the subscript 0 refers to the fracture, 1 refers to the first matrix volume, and 2 refers to the second matrix volume. The $x$ represents the unknown variable or variable pair. The one dimensional character of the matrix diffusion means that the second matrix node can only depend on the first matrix node. Therefore, the submatrix $\left[A_{20}\right]$ is empty. The fact that matrix nodes cannot communicate with matrix nodes in other computational cells means that the submatrices $\left[A_{21}\right]$ and $\left[A_{22}\right]$ are diagonal, therefore

$$
\left\{x_{2}\right\}=\left[A_{22}\right]^{-1}\left[-\left\{b_{2}\right\}-\left[A_{21}\right]\left\{x_{1}\right\}\right]
$$


where the inversion is trivial because $\left[A_{22}\right]$ is diagonal. Substituting this expression into the equation for the first matrix node gives

$$
\begin{aligned}
& {\left[A_{10}\right]\left\{x_{0}\right\}+\left[A_{11}\right]\left\{x_{1}\right\}+} \\
& \quad\left[A_{12}\right]\left[A_{22}\right]^{-1}\left[-\left\{b_{2}\right\}-\left[A_{21}\right]\left\{x_{1}\right\}\right]=-\left\{b_{1}\right\}
\end{aligned}
$$

Rearranging,

$$
\begin{aligned}
& {\left[A_{10}\right]\left\{x_{0}\right\}+\left[\left[A_{11}\right]-\left[A_{12}\right]\left[A_{22}\right]^{-1}\left[A_{21}\right]\right]\left\{x_{1}\right\}=} \\
& -\left\{b_{1}\right\}+\left[A_{12}\right]\left[A_{22}\right]^{-1}\left\{b_{2}\right\}
\end{aligned}
$$

or

$$
\left\{x_{1}\right\}=\left[\tilde{A}_{11}\right]^{-1}\left[\left\{\tilde{b}_{1}\right\}-\left[A_{10}\right]\left\{x_{0}\right\}\right]
$$

where

$$
\left[\tilde{A}_{11}\right]=\left[A_{11}\right]-\left[A_{12}\right]\left[A_{22}\right]^{-1}\left[A_{21}\right]
$$

and

$$
\left\{\tilde{b}_{1}\right\}=-\left\{b_{1}\right\}+\left[A_{12}\right]\left[A_{22}\right]^{-1}\left\{b_{2}\right\}
$$

The inversion and multiplications are trivial because of the diagonal nature of the matrices involved. Equation (67) may next be substituted into the equation for the fracture variables. Noting that $\left[A_{02}\right]$ is empty (the fracture can only communicate with the first matrix volume) gives

$$
\left[A_{00}\right]\left\{x_{0}\right\}+\left[A_{01}\right]\left[\tilde{A}_{11}\right]^{-1}\left[\left\{\tilde{b}_{1}\right\}-\left[A_{10}\right]\left\{x_{0}\right\}\right]=-\left\{b_{0}\right\} \text {. }
$$

Rearranging terms results in 


$$
\begin{gathered}
{\left[\left[A_{00}\right]-\left[A_{01}\right]\left[\tilde{A}_{11}\right]^{-1}\left[A_{10}\right]\right]\left\{x_{0}\right\}=} \\
-\left\{b_{0}\right\}+\left[A_{01}\right]\left[\tilde{A}_{11}\right]^{-1}\left\{\tilde{b}_{1}\right\} .
\end{gathered}
$$

Equation (71) consists of an augmented fracture matrix of the same form as the original fracture matrix $\left[A_{00}\right]$. The operations carried out only add a few percent to the solution time required to solve a single porosity system. After the solution of Equation (71) is obtained with the methods described in the GZSOLVE MMS, the solution in the fracture volume can be obtained by using Equations (65) and (67).

\subsubsection{Double Porosity / Double Permeability}

The Double Porosity / Double Permeability method is analogous to the Dual Porosity method described above with the exception that there is only one matrix node represented in the Double Porosity / Double Permeability method. The matrix node, however, can communicate globally to other matrix nodes. This leads to a system of equations of the form

$$
\left[\begin{array}{ll}
A_{00} & A_{01} \\
A_{10} & A_{11}
\end{array}\right]\left\{\begin{array}{l}
x_{0} \\
x_{1}
\end{array}\right\}=-\left\{\begin{array}{l}
b_{0} \\
b_{1}
\end{array}\right\}
$$

In this system of linear equations, the submatrices $A_{00}$ and $A_{11}$ are sparse and $A_{O I}$ and $A_{10}$ are diagonal. Currently this system of linear equations is solved directly, but research to improve the efficiency of solution is ongoing.

\subsubsection{Location}

When enabled the fracture models are called during the equation generation and solution phases of the simulation. This is the same place as shown for the Flow and Transport models in Fig. 1.

\subsubsection{Numerical Stability and Accuracy}

The same considerations that were discussed in Section 8.1 .8 for the Flow and Transport models are valid here.

\subsubsection{Alternatives}

Other approaches to modeling fractures include the equivalent continuum approach, in which the fracture and matrix properties are averaged, and the discrete fracture approach, in which the fractures are modeled as individual computational cells. Both of these methods are included in the model described in Section 8.1, "Flow and Energy Transport".

There has also been some effort to use a combination of numerical and analytic techniques. In this approach the matrix flow is represented with a one dimensional analytic expression. Because of the nonlinear nature of the solution, this approach has not been pursued. 


\subsection{Solute Transport - Reactive Transport and Particle Tracking Models}

\subsubsection{Purpose}

The purpose of the reactive transport and particle tracking models are to simulate the movement of tracer solutes traveling in either the liquid or gas phases. A variety of reactive transport capabilities are present in the models. To perform a reactive transport simulation, an initial description of each solute concentration in each phase, transport properties of the fluid and medium, and a specification of the adsorption model and parameters and any reaction models are required. The output consists of the final concentration of each solute in each phase.

\subsubsection{Assumptions and Limitations}

Solutes are assumed to be present in trace quantities, such that their presence does not impact the fluid properties or the computed flow fields. A related assumption is that chemical reactions do not enter into the energy balance through endothermic or exothermic reaction terms. If reactions take place between the fluid and solid phases (dissolution and precipitation), the transfer of mass is assumed to have no impact on the hydrologic properties of the medium.

Many other specific assumptions are built into the solute transport models related to the nature of the transport and chemical reaction behavior. These assumptions are treated in Section 8.3.3.

\subsubsection{Derivation}

\subsubsection{Reactive Transport Model}

The solute transport equations in the reactive transport model are not directly coupled to the heat and mass transfer system, but use the flow rates and temperatures obtained by the heat and mass transfer solution. The mass flux, $\bar{f}_{c}$, source (or sink) strength, $q_{c}$, and accumulation term, $A_{c}$, are defined as follows for a solute:

$$
\begin{aligned}
& \bar{f}_{c}=C_{v} \rho_{v} \bar{v}_{v}+C_{l} \rho_{l} \bar{v}_{l}, \\
& q_{c}=C_{v} q_{v}+C_{l} q_{l}, \\
& A_{c}=\phi\left(C_{v} S_{v} \rho_{v}+C_{l} S_{l} \rho_{l}\right) .
\end{aligned}
$$

The transport equation for a given solute is given by 


$$
\begin{gathered}
-\bar{\nabla} \cdot\left(C_{v} D_{m v} \bar{\nabla} P_{v}\right)-\bar{\nabla} \cdot\left(C_{l} D_{m l} \bar{\nabla} P_{l}\right)-\bar{\nabla} \cdot\left(D_{c v} \bar{\nabla} C_{v}\right)-\bar{\nabla} \cdot\left(D_{c l} \bar{\nabla} C_{l}\right)+ \\
q_{c}+\frac{\partial}{\partial z} g\left(C_{v} D_{m v} \rho_{v}+C_{l} D_{m l} \rho_{l}\right)+\rho_{r} \frac{\partial C_{R}}{\partial t}+\frac{\partial A_{c}}{\partial t}=0
\end{gathered}
$$

Here $C$ is the concentration of the solute. The term $\bar{\nabla} \cdot\left(D_{c} \bar{\nabla} C\right)$ is the dispersion term and $\rho_{r} \frac{\partial C_{r}}{\partial t}$ is an equilibrium sorption term (see section below for the formulation for sorbing solutes). $C_{r}$ represents the adsorption of the solute onto the porous media. In addition, the term $q_{c}$ includes the source or sink due to chemical reaction. The chemical reaction terms are discussed in more detail below in the section titled "Chemical Reaction Module."

Equation (76) is a general equation for a solute present in either the liquid or gas phases, or one that partitions between the liquid and gas. The model is capable of simulating any of these possibilities, as well as a solid species, for which only the accumulation and chemical reaction terms are present. Several solutes can be simulated simultaneously, and can interact with one another through the chemical reaction model. The transport terms can be set as a function of position, and there is no requirement that they be the same for all solutes present in a phase.

The next four subsections elaborate on various transport, sorption, and reaction features of the reactive transport model.

Dispersion Coefficients. The model uses a standard formulation for the dispersion coefficient, expressed as follows for the $\mathrm{x}$-direction

$$
D_{c l, x}=D_{A B}+\alpha_{d l, x} v_{l, x} \text {. }
$$

The Darcy velocity is computed from the solution of the fluid flow equation. The dispersivity $\alpha_{d}$ and the molecular diffusion coefficient $D_{A B}$ are properties of the medium, the fluid (liquid in the above equation) and the solute. Similar expressions are written for the $y$ - and $z$-directions.

Adsorbing Solutes. The general equilibrium model for adsorption of species onto the reservoir rock is given by (Polzer, et al. 1992) 


$$
C_{r}=\frac{\alpha_{1} C_{l}^{\beta}}{1+\alpha_{2} C_{l}^{\beta}}
$$

The parameters $\alpha_{1}, \alpha_{2}$, and $\beta$ are given in Table II along with the commonly used sorption isotherm models that can be derived from the equation. The parameters $\mathrm{K}_{d}, \Lambda, C_{\text {max }}, r_{b}$, and $r$ are the corresponding parameters associated with the sorption models as they are more commonly formulated. For example, when the linear, equilibrium sorption model is selected, the $\alpha_{1}$ parameter is the widely used $K_{d}$ parameter cited in sorption studies.

To solve the solute mass balance equation with equilibrium sorption, $C_{R}$ in Equation (76) is computed using Equation (78) to determine the mass of solute on the rock for a given fluid-phase concentration. Thus, $C_{R}$ is not actually present as a separate unknown in the mass balance.

\begin{tabular}{|c|c|c|c|c|}
\hline \multicolumn{5}{|c|}{ Table II. Sorption Isotherm Models $\ddagger$} \\
\hline \hline Model & Expression & $\alpha_{1}$ & $\alpha_{2}$ & $\beta$ \\
\hline Linear & $C_{r}=K_{d} C_{l}$ & $K_{d}$ & 0. & 1 \\
\hline Freundlich & $C_{r}=\Lambda C_{l}^{\beta}$ & $\Lambda$ & 0 & $0<\beta<1$ \\
\hline $\begin{array}{c}\text { Modified } \\
\text { Freundlich }\end{array}$ & $\frac{C_{r}}{C_{r, m a x}-C_{r}}=\Lambda C_{l}^{\beta}$ & $\Lambda C_{r, m a x}$ & $\Lambda$ & $0<\beta<1$ \\
\hline Langmuir & $C_{r}=\frac{r_{b} C_{l}}{1+r C_{l}}$ & $r_{b}$ & $r$ & 1 \\
\hline
\end{tabular}

Henry's Law Species. In contrast to a liquid only or vapor only species, all transport terms of Equation (76) are retained (both liquid and vapor). The vapor concentration is related to the liquid concentration assuming the equilibrium Henry's law equation

$$
C_{v}=\frac{M_{w} K_{H} C_{l}}{M_{v} P_{v}}
$$


where $M_{w}$ is the molecular weight of water, $M_{v}$ is the molecular weight of the vapor, $P_{v}$ is the gas pressure, and $K_{H}$ is the Henry's law coefficient. Temperature dependence of the Henry's Law constant is modeled using the following relation

$K_{H}=A_{H} \exp \left(\frac{\Delta H_{H}}{R\left(\frac{1}{298.16}-\frac{1}{T}\right)}\right)$

where $A_{H}$ and $\Delta H_{H}$ are model parameters, $R$ is the universal gas constant ( $\left.8.314 \times 10^{-3} \mathrm{~kJ} / \mathrm{mol}-\mathrm{K}\right)$, and $T$ is the temperature in degrees Kelvin. The units for $\Delta H_{H}$ are $\mathrm{kJ} / \mathrm{mol}$, and the units for $A_{H}$ and $K_{H}$ are $\mathrm{MPa}$ /liquid-mole-fraction.

An alternate formulation of the temperature dependence of the Henry's law coefficient is also available. It is included specifically to model the dissolution of $\mathrm{CO}_{2}$ into the liquid phase. The empirical correlation used to fit data for $\mathrm{CO}_{2}$ dissolution by Plummer and Busenberg (1982), after converting into the units required by FEHM, is

$K_{H}=\frac{10.1325 \rho_{l}}{M_{w} \hat{K}_{H}}$

where $\hat{K}_{H}=10^{\tilde{K}_{H}}$, and

$\tilde{K}_{H}=A_{H, 1}+A_{H, 2} T+\frac{A_{H, 3}}{T}+A_{H, 4} \log _{10} T+\frac{A_{H, 5}}{T^{2}}$.

Multiple, Interacting Solutes. Thus far, only the specification of an individual solute has been discussed. In the reactive transport model, chemical reactions involving one or more components can be specified with the following form:

$$
\begin{aligned}
& a_{1} B_{1}+a_{2} B_{2}+\ldots+a_{m} B_{m}= \\
& \quad a_{m+1} B_{m+1}+a_{m+2} B_{m+2}+\ldots+a_{n} B_{n}
\end{aligned}
$$

where the $a$ 's are the stoichiometric coefficients and the $B$ 's denote each solute present in a particular reaction (i.e., the $m$ th or $m+1$ th component). This relationship is formulated for each 
reaction being modeled, and a solute may be present in any number of reactions as either a reactant or a product.

The reactions may be specified either as kinetically controlled or equilibrium reactions. For a kinetically controlled reaction, the rate law governing each reversible reaction is specified as follows:

$$
\frac{\partial}{\partial t}\left[B_{j}\right]= \pm a_{j}\left\{k_{f o r} \prod_{i=1}^{m}\left[B_{i}\right]^{b_{i}}-k_{r e v} \prod_{i=m+1}^{n}\left[B_{i}\right]^{b_{i}}\right\}
$$

Here the square brackets [ ] denote concentration, the $b_{i}$ are exponents in the reaction rate or equilibrium equation (specified for every reactant in each reaction), and the forward and reverse reaction rate constants $k_{f o r}$ and $k_{r e v}$ are governed by the Arrhenius equation:

$$
k_{f o r}=A_{f o r} \exp \left(\frac{-E_{f o r}}{R T}\right) \text {. }
$$

In Equation (84), the stoichiometric coefficient $a_{j}$ pre-multiplying the rate law expression is negative if $B_{j}$ is a reactant, since it is being consumed in the reaction.

For equilibrium reactions, the following relationship is satisfied:

$$
K_{e q}=\frac{\prod_{i=m+1}^{n}\left[B_{i}\right]^{b_{i}}}{\prod_{i=1}^{m}\left[B_{i}\right]^{b_{i}}}
$$

where $K_{e q}$ is the equilibrium constant for the reaction. The temperature dependence of $K_{e q}$ can be expressed in two ways, similar to the specification of Henry's law constants above. In the first model, the van't Hoff relationship is used:

$$
K_{e q}=K_{e q, 25} \exp \left(\frac{\Delta H_{r x n}}{R\left(\frac{1}{298.16}-\frac{1}{T}\right)}\right)
$$


Alternatively, a formulation allowing simulation of the carbonate reaction system is included, which uses the following form:

$K_{e q}=10^{\hat{K}_{e q}}$

where

$\hat{K}_{e q}=A_{r x n, 1}+A_{r x n, 2} T+\frac{A_{r x n, 3}}{T}+A_{r x n, 4} \log _{10} T+\frac{A_{r x n, 5}}{T^{2}}$

In Equations (87) and (89), the temperatures are in degrees Kelvin.

For sorbing species, reaction may occur for solute in the fluid phase, in the sorbed phase, or both. For the modified Freundlich isotherm [Equation (78)], the total concentration used in the reaction rate law for the case of fluid and sorbed phase solute participating in the reaction is

$$
\left[B_{j}\right]_{T O T}=\left[B_{j}\right]+\left[B_{j}\right]_{\text {rock }}=\left[B_{j}\right]+\frac{\rho_{r} a_{1}\left[B_{j}\right]^{\beta}}{\rho_{p} \phi\left(1+a_{2}\left[B_{j}\right]^{\beta}\right)}
$$

where $\rho_{r}$ is the bulk rock density, $\rho_{p}$ is the fluid density, $\phi$ is porosity, and $a_{1}, a_{2}$, and $\beta$ are the sorption isotherm parameters. Effectively, the second term on the right hand side of Equation (90) is the equivalent concentration of the sorbed species if it were present in the fluid phase. The assumption that reactivity is identical for solute regardless of phase is valid for radioactive decay, but will certainly be incorrect for some chemical reactions. Thus, FEHMN provides an option whereby for each species in each reaction, the user may specify whether the reaction applies to solute in the fluid phase (concentration of $\left[B_{j}\right]$ ), solute in the sorbed phase [concentration given by the fraction on the right hand side of Equation (90)], or both. For two-phase flow, $\rho_{p}$ is replaced by $S_{p} \rho_{p}$, where $S_{p}$ is the saturation of the phase $(p)$ containing the solute.

For reactions involving a solid species, typically a zero order chemical reaction is assumed, though this is not required. The concentration of a solid is expressed in moles of species per $\mathrm{kg}$ rock, whereas, all other concentrations in the code are expressed in moles of species per $\mathrm{kg}$ of fluid. The model for solid reactions undergoing zero order reactions accounts for the degree of saturation when computing rates. When there is no solid 
present, a solution must be supersaturated (the rate of the reaction forming the solid must be greater than the rate of the reaction consuming the solid), or else the reaction is assumed to not take place.

Finally, when Henry's law species are specified as undergoing chemical reactions, it is assumed that the reaction takes place for solute in either the liquid or vapor phases, but not both. The user must specify which phase participates in the reaction. When it is desired that the reaction take place in both phases (say, for a radioactive decay reaction), the user must specify two reactions with identical rate expressions, one for the liquid borne portion of the solute and one for the vapor borne portion.

Solute Sources and Sinks. Solute sources or sinks are handled in a manner analogous to the fluid flow sources and sinks. If there is fluid flow out of the model domain (a fluid sink), the in-place solute concentration is used in the solute mass balance. For fluid entering the system, the solute concentration of the incoming fluid can be specified. Alternatively, the concentration at a node or nodes can be held at a fixed concentration. This boundary condition can be either a source or sink for solute, depending on the gradient in concentration at locations adjacent to the node at which the boundary condition is applied.

\subsubsection{Particle Tracking Model}

The particle tracking method developed in FEHM views the fluid flow computational domain as an interconnected network of fluid storage volumes. The description that follows is applicable for steady state flow fields; the variations in the method for treating transient flow systems are discussed later. The two steps in the particle tracking approach are: 1 ) determine the time a particle spends in a given cell, and 2) determine which cell the particle travels to next. These two steps are detailed below.

The residence time that a particle spends in a cell is governed by a transfer function describing the probability of the particle spending a given length of time in the cell. Thus, this particle tracking approach is called the "residence time transfer function" (RTTF) method. For a cumulative probability distribution function of particle residence times, the residence time of a particle in a cell is computed by generating a random number between 0 and 1 , and determining the corresponding residence time. If a large number of particles pass through the cell, the cumulative residence time distribution (RTD) of particles in the cell will be reproduced.

From the solution of the flow field in a numerical model, the mass of fluid in the computational cell, and the mass flow rate to or from each adjacent cell is obtained. In the simplest case, 
the residence time of a particle within each finite difference cell $\tau_{\text {part }}$ is given by

$$
\tau_{\text {part }}=\tau_{f}=\frac{M_{f}}{\sum \dot{m}_{\text {out }}}
$$

where $M_{f}$ is the fluid mass associated with the cell and the summation term in the denominator refers to the outlet mass flow rates from the cell to adjacent cells. In the absence of dispersion or other transport mechanisms, the transfer function is a Heaviside function that is unity at the fluid residence time $\tau_{f}$, since for this simple case all particles possess this residence time. Equilibrium, linear sorption is included by correcting the residence time by a retardation factor $R_{f}$, so that

$$
\begin{aligned}
& \tau_{\text {part }}=R_{f} \tau_{f}, \text { where } R_{f} \text { is given by } \\
& R_{f}=1+\frac{\rho_{b} K_{d}}{\phi S_{f} \rho_{f}} .
\end{aligned}
$$

In Equation (92), $K_{d}$ is the equilibrium sorption coefficient, $\rho_{b}$ is the bulk rock density, $\phi$ is the porosity, $S_{f}$.is the saturation of the phase in which the particle is traveling, and $\rho_{f}$ is the density of the fluid. Once again, in the absence of other transport processes, the transfer function is a Heaviside function.

Before discussing more complex examples of the RTTF method, the method for determining which cell a particle travels to after completing its stay at a given cell is outlined. The assumption that is consistent with the RTTF method is that the probability of traveling to a neighboring cell is proportional to the mass flow rate to that cell. Only outflows are included in this calculation; the probability of traveling to an adjacent node is 0 if flow is from that node to the current node. By generating a uniform random number from zero to one, the decision of which node to travel to is straightforward. Thus the particle tracking algorithm is: 1) compute the residence time of a particle at a cell using the RTTF method; and 2) send the particle to an adjacent cell randomly, with the probability of traveling to a given cell proportional to the mass flow rate to that cell.

The transfer function for transport processes such as dispersion are described now. Within a computational cell, it is assumed that one-dimensional, axial dispersion is valid. The transport 
equation and boundary conditions for the one-dimensional, advective-dispersion equation are

$$
\begin{array}{ll}
R_{f \partial t} \frac{\partial C}{\partial t}=D_{e f f} \frac{\partial^{2} C}{\partial x^{2}}-v \frac{\partial C}{\partial x}, \\
C=C_{\text {in }}, & \text { at } x=0 \text { and } \\
\frac{\partial C}{\partial x}=0, & \text { for } x \rightarrow \infty .
\end{array}
$$

In the equations above, $C$ is the concentration, $C_{i n}$ is the injection concentration, $v$ is the superficial flow velocity, and $D_{e f f}$ is the effective dispersion coefficient, given by $D_{\text {eff }}=\alpha v$, where $\alpha$ is the dispersivity of the medium. Here it is assumed that the flow dispersion component of $D_{\text {eff }}$ is large compared to the molecular diffusion coefficient $D_{A B}$. A nondimensional version of Equation (93) can be obtained using the following transformations: $\hat{C}=C / C_{i n}, \hat{x}=x / L, \hat{\theta}=R_{f} \nu t / L=R_{f} \tau_{f}$, where $L$ is the distance along the flow path where the concentration is being measured. Then, Equation (93) becomes

$\frac{\partial}{\partial \hat{\theta}} \hat{C}=P e^{-1} \frac{\partial^{2}}{\partial \hat{x}^{2}} \hat{C}-u \frac{\partial}{\partial \hat{x}} \hat{C}$

where $P e=v L / D_{\text {eff }}$ is the Peclet number. Alternatively, $P e=L / \alpha$. The solution to this equation and boundary conditions is given by Brigham (1974) as

$$
\hat{C}=\frac{1}{2}\left[\operatorname{erfc}\left(\frac{\sqrt{P e}(1-\hat{\theta})}{2 \sqrt{\hat{\theta}}}\right)+e^{P e} \operatorname{erfc}\left(\frac{\sqrt{P e}(1+\hat{\theta})}{2 \sqrt{\hat{\theta}}}\right)\right] \text {. }
$$

The use of this solution in the RTTF particle tracking method requires that the transport problem be advection dominated, so that during the time spent in a computational cell, solute would not tend to spread a significant distance away from that cell. Then, the approximate use of a distribution of times within the cell should be adequate. Quantitatively, the criterion for applicability is based on the grid Peclet number $P e_{g}=\Delta x / \alpha$, 
where $\Delta x$ is the characteristic length scale of the computational cell. Note that in contrast to conventional solutions to the advective-dispersion equations, coarse spatial discretization is helpful in satisfying this criterion, as long as the mesh spacing is small enough to provide an accurate flow solution. Highly dispersive transport invalidates the assumptions of the RTTF particle tracking technique. This is not viewed as a severe limitation of the method, since accurate solutions to the advective-dispersion equation are easily obtained by conventional finite difference or finite element techniques for this case. The niche filled by this new technique is in the solution of advection-dominated problems involving the movement of sharp concentration fronts.

For multi-dimensional flow systems, this method for simulating dispersion can be extended for the case of dispersion coefficient values aligned with the coordinate axes. For this case, the flow direction is determined by the vector drawn from the nodal position of the cell the particle traveled from to the current cell, and the dispersivity for this flow direction is given by

$$
\alpha=\frac{\Delta x \alpha_{x}+\Delta y \alpha_{y}+\Delta z \alpha_{z}}{L}
$$

The RTTF particle tracking technique cannot be formulated with a longitudinal and transverse dispersion coefficient model, since the flow rates between cells are defined, rather than the actual flow velocity at a position. For a dispersion model aligned to the flow direction, the particle tracking method such as that of Tompson and Gelhar (1990) or a conventional finite element or finite difference solution to the advective-dispersion equation should be used.

Matrix Diffusion. Matrix diffusion has been recognized as an important transport mechanism for fractured porous media (Neretnicks, 1980, Robinson, 1994). For many hydrologic flow systems, fluid flow is dominated by fractures, because of the orders of magnitude larger permeabilities in the fractures compared to the surrounding rock matrix. However, even when the fluid in the matrix is completely stagnant, solute can move into the matrix via molecular diffusion, resulting in a physical retardation of solute compared to pure fracture transport. This effect has recently been demonstrated at the laboratory scale by Reimus (1995), and at the field scale by Maloszewski and Zuber (1985).

To develop a transfer function for matrix diffusion, an idealized representation of the transport system must first be developed. Figure 5 shows the geometry of the model system used for this purpose. The geometry and flow system consists of equally spaced, parallel fractures, each of which transmits equal flow. Fluid in the rock matrix is stagnant. Transport in the fractures 
is governed by Equation (93) with an additional term $q_{f m}$ on the right hand side, given by

$$
q_{f m}=\left.\frac{2 \phi_{m a t} D_{A B}}{b_{f}} \frac{\partial C}{\partial y}\right|_{y=b / 2}
$$

where $\phi_{\text {mat }}$ is the matrix porosity and $b_{f}$ is the fracture aperture. Transport between the fracture and matrix is governed by the one-dimensional diffusion equation:

$$
R_{f, m} \frac{\partial C}{\partial t}=D_{A B} \frac{\partial^{2} C}{\partial y^{2}}
$$

where $R_{f, m}$ is the retardation coefficient for the matrix. The molecular diffusion coefficient is a function of the free diffusion coefficient of the solute in water and a tortuosity factor to account for the details of diffusion through the tortuous, fluid filled pore network. In this model, $D_{A B}$ is treated as the fundamental transport parameter, recognizing that it is a property of both the solute and the medium. Solutions to this transport problem depend on the nature of the boundary condition away from the fractures. An analytical solution is given by Tang, et al. (1981) for the semi-infinite boundary

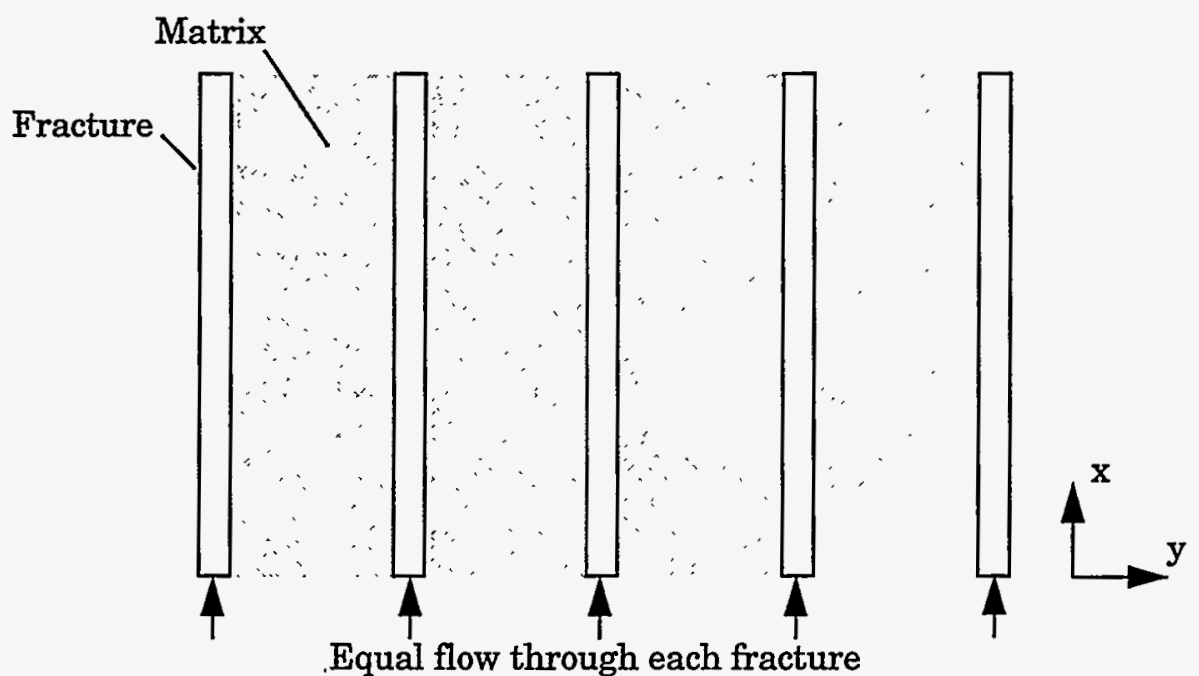

Figure 5. Model system used to formulated the residence time transfer function for matrix diffusion 
condition $\frac{\partial C}{\partial y}=0$ as $y \rightarrow \infty$. For the case of plug flow (no

dispersion) in the fractures, Starr, et al. (1985) show that the solution reduces to

$$
\hat{C}=\operatorname{erf}\left[\frac{\phi_{m a t} \tau_{f \sqrt{R_{f, m} D_{A B}}}}{b_{f} \sqrt{t-R_{f} \tau_{f}}}\right] .
$$

The semi-infinite boundary condition between fractures limits the validity of either of these solutions to situations in which the characteristic diffusion distance for the transport problem is small compared to the fracture spacing. However, as long as the solute has insufficient time to diffuse to the centerline between fractures, the solutions provided by Tang, et al. or Starr, et al. are valid to represent the transfer function for the particle tracking technique.

Although in principal the Tang, et al. solution could be used for the transfer function, its complex form makes it very inconvenient for rapidly computing particle residence times. Instead, a two-step process is used in which the residence time within the fracture is first computed using the transfer function for one-dimensional dispersion in Equation (97) without sorption. Then, the plug-flow equation with matrix diffusion and sorption (Equation (101)) is used with the value of the fracture residence time just determined to set the transfer function for the matrix diffusion component of the model. To use Equation (101) as a transfer function, a subroutine was developed to determine the inverse of the error function, that is, the value of $x_{d}$ for a given value of $y_{d}$, such that

$y_{d}=\operatorname{erf}\left(x_{d}\right)$. The numerical implementation of this method entails dividing the error function into piecewise continuous segments from which the value of $x_{d}$ is determined by interpolation. The use of the two-step approach is justified because of the principle of superposition, which allows the decoupling of the dispersive process in the fracture from the diffusive transport in the matrix.

Radioactive decay. Radioactive decay is important to many of the applications for which this model was developed, namely nuclear waste repository studies. Natural isotopes such as ${ }^{36} \mathrm{Cl}$ and ${ }^{14} \mathrm{C}$ also require the simulation of radioactive decay. This phenomenon can be treated by introducing the decay equation for an irreversible first order reaction:

$$
\hat{C}=\exp \left(-k_{R n} \tau_{a g e}\right)
$$


where $\tau_{\text {age }}$ is the particle age since entering the system, and $k_{R n}$ is the rate constant for radioactive decay, related to the radioactive decay half-life $\tau_{1 / 2}$ by $k_{R n}=0.693 / \tau_{1 / 2}$.

In this model, the concept of a fraction of a particle is used to incorporate radioactive decay into the calculation. The age of a particle, or time since entering the system, is used in Equation (102) to compute the fraction of the particles remaining at the current time. When concentration values are computed from the composite behavior of a large number of particles, this method accurately accounts for radioactive decay.

Particle Sources and Sinks. There are two methods for introducing particles into the flow system: 1) inject the particles with the source fluid entering the model domain, or 2) release the particles at a particular node or set of nodes. The first method is used to track injected fluid as it passes through the system. The number of particles entering with the source fluid at each cell is proportional to the source flow rate at that node. The method is the particle tracking equivalent to a constant solute concentration in the source fluid. For method 2, an equal number of particles are released at each node specified, regardless of the source flow rate. In either case, the model calls for the particles to be released over a specified time interval. The code then computes a starting time for each particle.

For fluid exiting the model domain, the model treats this flow as another outlet flow from the node. The decision of whether the particle leaves the system or travels to an adjacent node is then made on a probabilistic basis, just as though the fluid sink were another connected node. When a particle leaves the system, its sojourn through the model domain is completed; this fact is recorded as part of the statistics of the simulation.

Transient Flow Fields. When RTTF particle tracking method is implemented for a time varying fluid flow system, the approach is somewhat more complex but still tractable. Consider a numerical simulation in which a discrete time step is taken at time $t$, and a new fluid flow field is computed. In this model, transient flows are handled by treating the new fluid flow time $t_{n e w}$ as an intermediate time in the particle tracking calculation that the simulation must stop at. The fate of all particles is tracked from time $t$ to time $t_{n e w}$ assuming that the flow field is constant over this time interval. When the simulation reaches $t_{n e w}$, the position of the particle is recorded, along with the fractional time remaining for the particle at the cell, and the randomly generated y-coordinate of the transfer function used for that particle in the cell. When the new fluid flow solution is established, the process continues, but the remaining residence time for a particle is the time determined 
from the new transfer function times the fractional time remaining in the cell.

Another transient effect that must be considered is that the sum of the outlet mass flow rates $\sum \dot{m}_{\text {out }}$ in Equation (91) does not necessarily equal the sum of the inlet mass flow rates. When there is net fluid storage in a cell, the particle tracking algorithm uses the sum of the inlet flow rates in Equation (91), whereas Equation (91) itself is used when there is net drainage of fluid.

\subsubsection{Applications}

For transport calculations using either the reactive transport or particle tracking models, the validity of the solution depends first on the accuracy of the flow equations. In addition, the reliability of the transport parameters is also a factor in the representativeness of any transport simulation.

For the reactive transport model, the issue of numerical accuracy is extremely important to the usefulness of the results. The accuracy may be evaluated by solving the same problem using different size grids and evaluating the change in the solution. The major source of numerical errors for transport solutions is anticipated to be the numerical dispersion resulting from the upwinding of the advection term. Alternatively, the particle tracking module can be used for advection-dominated problems to provide a solution that can be compared to the reactive transport results.

The primary applications of the particle tracking model are:

- To generate transport solutions that are able to track sharp fronts in concentration without numerical dispersion, thereby allowing results from the reactive transport model to be evaluated for numerical accuracy;

- To allow fluid pathways to be mapped out visually using particles that follow the fluid;

- To provide a transport solution for a solute that diffuses into the rock matrix;

- To compile statistics on the distribution of fluid ages present at a given location.

Several limitations of the particle tracking model should be noted. The particle tracking method produces a transport solution that is free of numerical dispersion when flow is predominantly aligned with the fluid flow finite element grid. Grid orientation effects may be present when flow travels diagonally across the grid. The dispersion model extends the transport solution beyond a simple "plug flow" transport model, but the RTTF method is only valid for advection-dominated problems. In regions of a model domain where the grid Peclet number is less than about 1 , the method produces inaccurate results. Finally, the matrix diffusion method is valid only if the solute has insufficient time to diffuse fully between fractures during the time scale of a simulation.

\subsubsection{Numerical Method Type}

For the reactive transport model, the approximation of the partial differential equations for solute transport parallels exactly the theory 
outlined for the solution of the flow and energy transport equations in Section 8.1.6. The concentrations of all solutes must be solved simultaneously, since the concentrations are coupled through the kinetic or equilibrium reaction terms. The code employs an option to solve multiple solute concentrations directly using the multiple degree-of-freedom equation solver for up to four solutes. When more than four solutes are present, an iterative procedure is required. This method is outlined in detail in Section 8.3.6.

The RTTF particle tracking method is a Lagrangian numerical method that employs transfer functions to compute particle residence times in each cell. Thus the time a particle spends in a cell, as well as the decision of which adjacent cell to travel to next, are determined probabilistically.

\subsubsection{Derivation of Numerical Model}

\subsubsection{Reactive Transport Model}

Since many aspects of the reactive transport numerical methods parallel the development of the fluid and energy transport numerical method, only the parts of the development that are unique to solute transport are outlined here. Internal to the code, the chemical reaction terms of the solute mass balance equations are always formulated as kinetic expressions with forward and reverse rate terms. For kinetically controlled reactions, these rate terms are the two product terms of Equation (84). Equilibrium reactions use the fact that at equilibrium the forward and reverse rates are equal, so that $K_{e q}=k_{f o r} / k_{r e v}$. Forward and reverse rate constants are forced to be in the correct ratio to simulate equilibrium, and as long as the rate constants are high enough, equilibrium is approximated. Of course, it is not known a priori what values to use for the rate constants. If the values are too low, equilibrium behavior is not approximated. A less obvious consideration is that if the values are too high, the rate terms in Equation (76) overwhelm the transport terms in the mass balance, and the reactive transport problem is not well-posed: the transport part of the mass balance gets lost in the solution of the equations.

To circumvent these problems, on the first solute time step the model starts at a relatively low value for the forward rate constant, computes the corresponding reverse rate constant consistent with the equilibrium constant for the reaction, solves the reactive transport problem, then performs a check to ensure that equilibrium is approximated everywhere in the model domain. The check is

$$
\gamma_{r x n}=1-a b s\left(\frac{k_{r e v} \prod_{i=m+1}^{n} B_{i}^{b_{i}}}{k_{f o r} \prod_{i=1}^{m} B_{i}^{b_{i}}}\right)<\gamma_{t o l}
$$


where $\gamma_{\text {tol }}$ is a user defined tolerance parameter defining how close to equilibrium to force each reaction. Comparing Equations (103) and (86), and making use of the fact that $K_{e q}=k_{f o r} / k_{r e v}$ at equilibrium, the value $1-\gamma_{r x n}$ can be seen as the ratio of the equilibrium quotient (the right hand side of Equation (86)) to the equilibrium constant. Setting $\gamma_{t o l}$ to, say, 0.01 forces this ratio to 0.99 , or roughly speaking, $99 \%$ of the way to equilibrium. If the check is not satisfied at all positions, the minimum value $1-\gamma_{r x n}$ is found, and the forward rate constant is multiplied by $\gamma_{r x n} / k_{f a c t}$, where $k_{f a c t}$ is a user defined parameter (assumed to be less than 1) that sets the rate at which the rate constants are increased to approach equilibrium behavior. Alternatively, $k_{f a c t}$ can be chosen to be a direct multiplier to the current forward rate constant, in which case the value is set greater than 1.

In either case, the process of solving the entire reactive transport system is repeated with higher and higher rate constants until Equation (103) is satisfied for all equilibrium reactions at all positions. In portions of the model domain where concentrations are low, it is possible that the reaction rates are low or even 0 even when equilibrium behavior is specified. The model can be made to skip the equilibrium check of Equation (103) when the forward rate (the denominator in Equation (103)) is less than a user specified reaction tolerance parameter, called $r_{f, t o l}$. When a new time step is taken, the rate constants determined previously are used to restart the process. These rate constants will usually be sufficient to assure equilibrium behavior at subsequent time steps, but the equilibrium check is still performed, and rate constants increased if necessary.

The system of equations representing the mass balance for each solute results in a coupled system of $\mathrm{N}_{\text {sol }} \times \mathrm{N}_{\text {eq }}$ equations. When kinetics are rapid compared to transport, either because the rate constants are set large or the equilibrium reaction option is chosen, the solution technique must be quite robust. The multiple degree-of-freedom solver naturally handles this sort of strongly coupled system of equations. However, the current solver handles up to four degrees-of-freedom (in this case, four coupled solutes). To solve for more than four solutes, an iterative procedure has been implemented in which the solutes are placed into groups of up to four solutes. The code solves the equations group by group. When a solute is not present in a group, the current values of concentrations are used in computing reaction rate terms, but those concentrations are not unknowns at that particular step of the solution. 
Since the calculation of concentrations in groups falling later in the sequence may impact the mass balance of solutes already solved for, the entire system is not necessarily converged after all groups are solved. An outer iterative loop over all groups is traversed until the residuals of all equations are low. At this point the entire system of equations is solved to the specified tolerance, and a new time step is taken.

\subsubsection{Particle Tracking Model}

All aspects of the numerical model for particle tracking are discussed in Section 8.3.3.2.

\subsubsection{Location}

The implementation sequence for either the Reactive Transport Model or the Particle Tracking Model is illustrated in Figure 1. The two models cannot be run simultaneously in the current version of FEHMN. After a heat and mass transfer time step is taken and the flow and temperature fields are determined, the solute transport solution is computed from the previous heat and mass time to the current time. The flow field used for the transport calculations are assumed to be unchanging during this time.

\subsubsection{Numerical Stability and Accuracy}

Reactive Transport Model. As in the heat and mass transfer solution discussion (Section 8.1.8), nonlinearities can give rise to problems with stability of the solution. The formulation of the problem as a fully coupled, implicit solution maximizes the likelihood of obtaining a stable, accurate solution. Accuracy is also intimately tied to the grid discretization, time step, and dispersion coefficients of the solutes. Advection dominated transport with low dispersion coefficients is well known to be difficult to simulate accurately with finite difference or finite element techniques. Testing the solution against the results of a calculation with smaller grid spacings and time steps is one way to assess the level of numerical dispersion. Another way is to compare the solution to a particle tracking simulation, which is designed to minimize numerical inaccuracies.

Particle Tracking Model. The accuracy of a RTTF particle tracking should be evaluated using the following considerations:

- The dispersion coefficient must be set high enough to avoid grid Peclet numbers less than 1 ; in fact, the code sets the Peclet number of a cell to 1 for any value lower than 1 .

- Diffusion into the rock matrix must be slow enough that the solute has insufficient time to diffuse fully to the centerline between fractures.

- If the velocity vectors are not aligned with the finite element grid, some inaccuracies due to grid orientation effects are to be expected.

- The number of particles in the simulation must be sufficient to minimize errors induced by statistical fluctuations.

\subsubsection{Alternatives}

Reactive Transport Model. Many different numerical formulations of the reactive transport problem are possible. A review of these methods was performed by Yeh and Tripathi (1989). These models differ in the number of species that can be simulated, and the nature of the chemical reactions 
that can be simulated. When equilibrium is assumed for all reactions, the reaction apart of the problem can effectively be decoupled from the transport, and considerable simplification results. For combined kinetic and equilibrium formulations, Friedly and Rubin (1992) have shown that similar simplifications are possible. Most models presented in the literature that use sophisticated chemical sub-models are restricted to simplified flow geometries and flow physics or require a flow solution as input, and the number of grid points that can practically be simulated is small.

The reactive transport model developed here was specifically designed for use in the context of large-scale two- and three-dimensional simulations. It was assumed that in the near future, computational resources would be insufficient to handle a large number of chemical species for a large-scale problem of many thousands of grid points. Therefore, the model development assumed that information from other sources (geochemical codes, literature data for a few key reactions and species) could be abstracted and distilled into a relatively small number of interacting solutes. Given this assumption, the logical method of solution was to utilize the multiple degree-of-freedom solution technology that is at the center of the FEHMN code. Alternative techniques such as those referred to above will be evaluated and incorporated into future versions of FEHMN, as needed.

Particle Tracking Model. The RTTF particle tracking modeling approach in FEHMN differs from most groundwater particle tracking algorithms reported in the literature [e.g. Tompson and Gelhar (1990), Lu (1994)]. These methods require that the velocity vector be resolved accurately at each location in the model domain. This usually involves an interpolation method to obtain the velocity at any position needed based on the values computed from a flow simulation (at cell faces or nodes, for example). Then, the algorithm consists of marching forward in small time steps, computing the trajectory and new location of the particle at the new time. Equilibrium, linear sorption is modeled by introducing a retardation factor to reduce the particle velocity. Dispersion is handled using a random walk approach that displaces the particle a certain amount during each time step, so that the particle samples a different velocity field than it would have in the absence of dispersion.

By contrast, the approach used in the FEHMN particle tracking algorithm uses the fluid mass fluxes from node to node as the basis for moving particles. These are the quantities that are actually known in integrated finite difference and finite element calculations, while the velocity vectors are interpolated results. Thus the implementation of the RTTF technique in an existing code like FEHMN is straightforward. Another practical advantage is that the computations are extremely fast: simulations with several million particles are practical using conventional workstations. One compromise in the approach is the limitation to advection dominated transport systems. This was thought to be a reasonable compromise, especially in the context of a code that already has a reactive transport module that easily handles systems with high dispersion coefficients. 


\subsection{Constitutive Relationships}

\subsubsection{Purpose}

The densities, viscosities, and enthalpies of water, water vapor, and air are required for the simulation of the Flow and Transport in a geologic repository. These constitutive relations depend on temperature and pressure. To be computationally efficient, the form of these relations must be easy to compute and accurate. To satisfy these needs rational polynomial fits to the National Bureau of Standards Steam Tables are used. The models require the pressure and temperature of a node as input and output the densities, viscosities, and enthalpies of the phases.

\subsubsection{Assumptions and Limitations}

At present, several fits of the data are available to the user. These allow usage of the relations for temperatures up to $360^{\circ} \mathrm{C}$ and pressures up to $110 \mathrm{MPa}$. If the variable exceeds the limits of the data, the FEHMN code will restart the timestep with a smaller time step size and try to keep the variable within the bounds of the data.

\subsubsection{Derivation}

Pressure and Temperature Dependent Fluid Properties. A porous flow simulator, such as FEHMN, with heat and mass transfer capabilities requires the functional dependence of the phase densities, the phase enthalpies, and the phase viscosities on temperature $(T)$ and pressure $(P)$. Because FEHMN is an implicit code which uses a Newton-Raphson iteration, derivatives of the thermodynamic functions with respect to $P$ and $T$ are also required.

Rational function approximations are used to estimate the thermodynamic variables in FEHMN where the rational functions are a ratio of polynomials. Complete polynomials of order three are used in both the numerator and denominator. For example, the density is approximated as

$$
\rho(P, T)=\frac{Y(P, T)}{Z(P, T)}
$$

where

$$
\begin{gathered}
Y(P, T)=Y_{0}+Y_{1} P+Y_{2} P^{2}+Y_{3} P^{3}+Y_{4} T+Y_{5} T^{2}+ \\
Y_{6} T^{3}+Y_{7} P T+Y_{8} P^{2} T+Y_{9} P T^{2}
\end{gathered}
$$

and

$$
\begin{gathered}
Z(P, T)=Z_{0}+Z_{1} P+Z_{2} P^{2}+Z_{3} P^{3}+Z_{4} T+Z_{5} T^{2}+ \\
Z_{6} T^{3}+Z_{7} P T+Z_{8} P^{2} T+Z_{9} P T^{2} .
\end{gathered}
$$


This type of relationship has been shown by Zyvoloski and Dash (1991) to provide an accurate method for determining parameter values over a wide range of pressures and temperatures, as well as allowing derivatives with respect to pressure and temperature to be computed easily.

Polynomial coefficients were obtained by fitting data from the National Bureau of Standards OSRD database 10, the database used for the NBS/ NRC Steam Tables (Harr, et al. 1984). The data fits result in errors less than one percent and often less than 0.1 percent. Two sets of coefficients are used in FEHMN one over the ranges $0.001 \leq P \leq 110.0 \mathrm{MPa}$ and $0.001 \leq T \leq 360^{\circ} \mathrm{C}$, and the other, for low pressures, with the ranges $0.001 \leq P \leq 20.0 \mathrm{MPa}$ and $0.5 \leq T \leq 360^{\circ} \mathrm{C}$. Polynomial coefficients for the enthalpy, density, and viscosity functions are given in Tables Table III, Table IV, and Table V, respectively, of the Appendix.

Pressure as a function of saturation temperature / Temperature as a function of saturation pressure. The equation for the saturation line is important for the determination of the phase state of the liquid vapor system. The saturation line may be described in a water only system as the pressure above which boiling occurs. In a mixture of air or other noncondensible gas, the saturation line is simply the partial pressure of water or the vapor pressure of water. Rational function approximations are also used for the saturation line equations:

$$
P_{v}(T)=\frac{Y(T)}{Z(T)}, T\left(P_{v}\right)=\frac{Y\left(P_{v}\right)}{Z\left(P_{v}\right)}
$$

where

$$
Y(X)=Y_{0}+Y_{1} X+Y_{2} X^{2}+Y_{3} X^{3}+Y_{4} X^{4}
$$

and

$$
Z(X)=Z_{0}+Z_{1} X+Z_{2} X^{2}+Z_{3} X^{3}+Z_{4} X^{4}
$$

$\mathrm{X}$ represents temperature or pressure in the respective relationships. Polynomial coefficients for the saturation functions are given in Table Table VI of the Appendix.

FEHMN also allows for the inclusion of a vapor pressure lowering term which may be important in situations where high capillary forces are present. The modified vapor pressure is given by:

$$
P_{v}^{*}\left(T, P_{c a p}\right)=P_{v}(T) \exp \left(\frac{P_{c a p}}{\rho_{l} R^{*}(T+273.15)}\right)
$$


where $P_{v}^{*}$ is the new vapor pressure of water, $P_{c a p}$ is the capillary pressure, and $R^{*}$ is the gas constant divided by the molecular weight of water.

Properties of Air and Air/Vapor Mixtures. Appropriate thermodynamic information for air and air/vapor mixtures are provided. The density of air is assumed to obey the ideal gas law. Using atmospheric conditions as the reference state

$$
\rho_{a}=1.292864\left(\frac{273.15}{T+273.15}\right)\left(\frac{P_{a}}{0.101325}\right)
$$

where $\rho_{a}$ has the units $\mathrm{kg} / \mathrm{m}^{3}, T$ is in ${ }^{\circ} \mathrm{C}$, and $P$ is in $\mathrm{MPa}$. The mixture density is given by

$$
\rho_{v}=\rho_{v, w}+\rho_{a}
$$

where $\rho_{v, w}$ is the density of water vapor.

The enthalpy of air is specified as a function of temperature only

$$
h_{a}=c_{p a}\left(T \cdot 10^{-6}\right)
$$

where

$$
c_{p a}=1003.7+0.025656 T+0.00045457 T^{2}-2.7107 \times 10^{-7} T^{3},
$$

$h_{a}$ is the enthalpy of air (MJ/kg), and $c_{p a}$ is the heat capacity of air (MJ/kg ${ }^{\circ} \mathrm{C}$ ). The parameters in Equation (114) were obtained by regression of a more complex correlation found in Sychev, et al. (1988). The mixture enthalpy for the vapor phase is

$$
h_{v}=h_{v, w}\left(1-\eta_{v}\right)+h_{a} \eta_{v}
$$

where $h_{v, w}$ is the enthalpy of steam and $\eta_{v}$ is the fraction by mass of air in the vapor phase. The mixture enthalpy of the liquid phase is given by:

$$
h_{l}=h_{l, w}\left(1-\eta_{l}\right)+h_{a} \eta_{l}
$$

where $h_{l, w}$ is the enthalpy of liquid water and $\eta_{l}$ is the mass fraction of air in the liquid phase.

Assuming ideal gas behavior, the mass fraction of air in the vapor phase may be expressed as 


$$
\eta_{v}=\frac{\rho_{a}}{\rho_{v}}
$$

The mass fraction of air in the liquid phase is assumed to obey Henry's law or

$$
\eta_{l}=K_{H, a} P_{a}
$$

where $K_{H, a}$ is the Henry's law constant for air $\left(K_{H, a}=1.611 \times 10^{-4} \mathrm{~Pa}^{-1}\right)$ and $P_{a}$ is the partial pressure of air.

The viscosity of the vapor phase is assumed to be a linear combination of the air viscosity and the water viscosity

$$
\mu_{v}=\mu_{v, w}\left(1-\eta_{v}\right)+\mu_{a} \eta_{v}
$$

where $\mu_{v, w}$ is the steam viscosity and is obtained from steam data. The viscosity of air is assumed constant

$$
\mu_{a}=1.82 \times 10^{-8} \frac{\mathrm{N} \cdot \mathrm{s}}{\mathrm{m}^{2}}
$$

The liquid phase viscosity is assumed to be independent of the amount of dissolved air and is obtained from a rational function approximation like those specified above.

Relative Permeability and Capillary Pressure Functions. Relative permeabilities and capillary pressures can be strong functions of saturation. Several well known relative permeability functions are available to the user. They are the simple linear functions, the Corey (1954) relationships, and the van Genuchten (1980) functions. Composite relative permeability curves, as described by Klavetter and Peters (1986), are also a user option.

The linear functions are given by

$$
R_{l}= \begin{cases}0, & S_{l} \leq S_{l r} \\ \frac{S_{l}-S_{l r}}{S_{l \max }-S_{l r},} & S_{l r}<S_{l}<S_{l \max } \\ 1, & S_{l} \geq S_{l \max }\end{cases}
$$




$$
R_{v}= \begin{cases}0, & S_{v} \leq S_{v r} \\ \frac{S_{v}-S_{v r}}{S_{v \max }-S_{v r}}, & S_{v r}<S_{v}<S_{v \max } \\ 1, & S_{v} \geq S_{v \max }\end{cases}
$$

where $S_{l r i}$ is residual liquid saturation, $S_{v r}$ is residual vapor saturation, $S_{l \max }$ is maximum liquid saturation, and $S_{v \max }$ is maximum vapor saturation.

The Corey relative permeability functions are given by

$$
\begin{aligned}
& R_{l}=\hat{S}_{l}^{4}, \\
& R_{v}=\left(1-\hat{S}_{l}\right)^{2}\left(1-\hat{S}_{l}^{2}\right)
\end{aligned}
$$

where $\hat{S}_{l}=\frac{S_{l}-S_{l r}-S_{v r}}{1-S_{l r}-S_{v r}}$ and $S_{l r}$ and $S_{v r}$ are the residual liquid and vapor saturations respectively.

The van Genuchten relative permeability functions are described by the following formulae:

$$
\begin{aligned}
& R_{l}=\left\{\begin{array}{cc}
{\left[1.0-\left(1.0-\hat{S}^{\frac{1}{\lambda}}\right)^{\lambda}\right]^{2} \sqrt{\hat{S},}} & \hat{S}_{l}<S_{l \max } \\
1.0, & \hat{S}_{l} \geq S_{l \max }
\end{array}\right. \\
& R_{v}=1.0-R_{l}
\end{aligned}
$$

where $\hat{S}=\frac{S_{l}-S_{l r}}{S_{l \max }-S_{l r}}$ and $\lambda=1-\frac{1}{n}$, where $n$ is an experimentally determined parameter.

$R_{l}$ and $R_{v}$ are restricted by the requirement that $0.0 \leq R_{l} \leq 1.0$ and $0.0 \leq R_{v} \leq 1.0$. The relative permeability functions are truncated to the appropriate value if the these conditions are violated.

The capillary functions considered are the linear function and the van Genuchten capillary pressure model. Our terminology follows that of Pruess (1987).

The linear capillary function model is given by the following equations 


$$
P_{\text {cap }}=\left\{\begin{array}{lc}
P_{\text {capmax }}, & S_{l} \leq S_{l r} \\
P_{\text {capmax }} \frac{S_{l \max }-S_{l}}{S_{l \max }-S_{l r},} & S_{l r}<S_{l}<S_{l \max } \\
0.0, & S_{l} \geq S_{l \max }
\end{array}\right.
$$

where $P_{\text {capmax }}$ is the maximum capillary pressure, $S_{l r}$ is the residual liquid saturation, and $S_{l m a x}$ is the maximum liquid saturation. The restriction $S_{l \text { max }}>S_{l r}$ is also necessary.

The van Genuchten functions (van Genuchten, 1980) for capillary pressure are described by the following equations

$$
P_{\text {cap }}= \begin{cases}P_{\text {capmax }}, & P_{\text {cap } 1} \geq P_{\text {capmax }} \\ P_{\text {cap } 1}, & P_{\text {cap } 1}<P_{\text {capmax }} \\ 0.0, & S_{l} \geq S_{l \max }\end{cases}
$$

where $P_{c a p 1}=P_{0}\left[\hat{S}^{-\frac{1}{\lambda}}-1.0\right]^{1.0-\lambda}, \hat{S}=\frac{S_{l}-S_{l r}}{S_{l m a x}-S_{l r}}$ and $P_{0}=\frac{1.0}{\alpha_{G}}$, and $\lambda=1-\frac{1}{n}$, where $n$ and $\alpha_{G}$ are experimentally determined parameters.

The van Genuchten capillary pressure curves approach an infinite value as $S_{l}$ approaches 0 and 1 . This requires the use of extrapolation techniques. At low saturations both linear and cubic fits are available. At high saturations a linear fit is used.

Stress Dependent Properties. Often it is necessary to accommodate changes in the rock porosity and permeability due to changes in effective stress caused by temperature and pore fluid pressure changes. A linear and nonlinear model are incorporated in the code for this purpose.

The linear pore pressure model for porosity is given by

$$
\phi=\phi_{0}+\left(1-\phi_{0}\right)\left(c_{r}-c_{g}\right)\left(P-P_{0}\right)
$$

where $\phi$ is the porosity at pressure $P, \phi_{0}$ is the porosity at pressure $P_{0}, c_{r}$ is the pore volume compressibility of the rock, and $c_{g}$ is the compressibility of the matrix grain material.

The nonlinear model of fracture porosity (Gangi, 1978, Appendix) is given by

$$
\phi=\phi_{0}\left[1-\left(\frac{P_{c}}{P_{x}}\right)^{m}\right]
$$


and

$$
P_{c}=\sigma-P-\alpha E \Delta T
$$

where $P_{c}$ is the closure stress, $\sigma$ is the in situ stress (assumed isotropic), $\alpha$ is the coefficient of thermal expansion of the rock, $E$ is Young's modulus, $\Delta T$ is the temperature change of the rock, and $P_{x}$ and $m$ are parameters in the model.

For either case the effect of stress and temperature changes on permeability are modeled with

$$
k=k_{0}\left(\frac{\phi}{\phi_{0}}\right)^{3}
$$

where $k_{0}$ is the permeability at porosity $\phi_{0}$.

\subsubsection{Application}

The Constitutive Relationships discussed in Section 8.4 describe parameters that are used in the models described in previous sections. The discussion provided in Section 8.1.4 is also applicable here.

\subsubsection{Numerical Method Type}

The Newton Raphson method is used to calculate saturation temperature as a function of pressure. The method has been previously described in Section 8.1.6.

\subsubsection{Derivation of Numerical Model}

The relative permeability and capillary functions represent the most nonlinear parts of FEHMN and special consideration has been given them. A procedure similar to that used by Nitao (1988) is used to restrict the van Genuchten capillary function, Equation (128), to finite values when approaching zero saturation. The procedure is simple. At a low saturation, usually input by the user, the van Genuchten functions are replaced with linear fits that match the van Genuchten function at the specified saturation value and attain a maximum value, usually twice the value at the specified saturation, at zero saturation. This new capillary pressure is then used in the calculation of the relative permeability. The formulation in FEHMN differs from Nitao's implementation in that it uses a cubic spline fit to match both the value and the slope at the specified saturation. At zero saturation the coefficients of the spline are adjusted so a zero slope and a zero second derivative is achieved. This assures a monatomically increasing function for the capillary pressure.

\subsubsection{Location}

The constitutive relationships are used to obtain the parameters that define the Flow and Energy Transport Equations. Referring to Fig. 1, the box labeled 'Get thermodynamic parameters' represents calls to routines that form the constitutive relationships. 


\subsubsection{Numerical Stability and Accuracy}

The formulation of the constitutive relationship is directly related to the overall accuracy of the FEHMN application. The accurate formulation of the water properties described in Section 8.4 .3 was motivated by the need to have accuracy combined with computability. The discussion in Section 8.4.6 showed the need to have continuous and finite values of the constitutive functions. The authors believe there is still much work to be done in the area of extending the range of the functions as well as finding representations that will allow better convergence of the Newton Raphson iteration.

\subsubsection{Alternatives}

FEHMN uses analytic derivatives of the constitutive relationships described in Section 8.4. The TOUGH code described by Pruess (1987) and the variant of TOUGH used by Nitao (1988) use numerical differences of the fluid and energy balance equations in the Newton Raphson iteration. Both of the methods have merit. The numerical derivative approach allows for possibly faster incorporation of new fluid physics models while the analytic derivative approach uses less iterations on tested problems (Reeves, 1993).

The functional representation of the constitutive models could be replaced by a tabular formulation. Several available codes have used tabular input for capillary and relative permeability data. FEHMN will also incorporate tabular representations in future versions.

\subsection{EXPERIENCE}

The FEHMN computer code and its predecessors have been used on a wide variety of problems ranging from geothermal to environmental remediation to radioactive transport. When used in conjunction with its available grid generation package and post processing tools it has been a successful tool for modeling very complex geological settings and coupled fluid processes. When benchmarked against other codes it has been shown to be extremely competitive (Reeves, 1993). 


\subsection{APPENDIX}

\begin{tabular}{|c|c|c|c|c|c|}
\hline \multicolumn{6}{|c|}{ Table Ill. Polynomial Coefficients for Enthalpy Functions } \\
\hline & & Liquid & Vapor & Liquid & Vapor \\
\hline \multirow{10}{*}{ 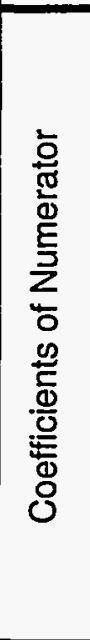 } & $Y_{0}$ & $0.25623465 \mathrm{e}-03$ & $0.31290881 e+00$ & $-0.28892688 \mathrm{e}-04$ & $0.49023415 e+00$ \\
\hline & $Y_{1}$ & $0.10184405 e-02$ & $-0.10000000 e+01$ & $0.10155128 \mathrm{e}-02$ & $-0.10000000 e+01$ \\
\hline & $Y_{2}$ & $0.22554970 \mathrm{e}-04$ & $0.25748596 e-01$ & $0.38182267 e-04$ & $0.24474474 \mathrm{e}-01$ \\
\hline & $Y_{3}$ & $0.34836663 e-07$ & $0.38846142 e-03$ & $0.29406408 \mathrm{e}-06$ & $0.23476073 e-03$ \\
\hline & $Y_{4}$ & $0.41 .769866 e-02$ & $0.11319298 \mathrm{e}-01$ & $0.42068671 \mathrm{e}-02$ & $0.86459576 \mathrm{e}-02$ \\
\hline & $Y_{5}$ & $-0.21244879 e-04$ & $0.20966376 e-04$ & $-0.26722745 e-04$ & $0.38256791 e-04$ \\
\hline & $Y_{6}$ & $0.25493516 \mathrm{e}-07$ & $0.74228083 e-08$ & $0.39965615 \mathrm{e}-07$ & $0.19190905 \mathrm{e}-07$ \\
\hline & $Y_{7}$ & $0.89557885 e-04$ & $0.19206133 e-02$ & $0.14983417 e-03$ & $0.16237610 \mathrm{e}-02$ \\
\hline & $Y_{8}$ & $0.10855046 \mathrm{e}-06$ & $-0.10372453 e-03$ & $0.11199162 \mathrm{e}-05$ & $-0.74126396 e-04$ \\
\hline & $Y_{g}$ & $-0.21720560 e-06$ & $0.59104245 e-07$ & $-0.44963038 e-06$ & $-0.78086106 e-06$ \\
\hline \multirow{10}{*}{ 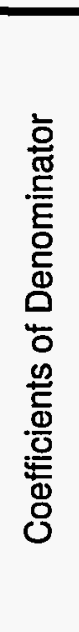 } & $\overline{Z_{0}}$ & $0.10000000 e+01$ & $0.12511319 e+00$ & $0.10000000 e+01$ & $0.19602927 \mathrm{e}+00$ \\
\hline & $Z_{I}$ & $0.23513278 e-01$ & $-0.36061317 e+00$ & $0.38028489 e-01$ & $-0.35954866 e+00$ \\
\hline & $Z_{2}$ & $0.48716386 e-04$ & $0.58668929 \mathrm{e}-02$ & $0.32800006 e-03$ & $0.54884993 e-02$ \\
\hline & $Z_{3}$ & $-0.19935046 e-08$ & $0.99059715 e-04$ & $0.38164755 \mathrm{e}-07$ & $0.58496026 \mathrm{e}-04$ \\
\hline & $Z_{4}$ & $-0.50770309 e-02$ & $0.44331611 \mathrm{e}-02$ & $-0.62817403 e-02$ & $0.33114850 \mathrm{e}-02$ \\
\hline & $Z_{5}$ & $0.57780287 e-05$ & $0.50902084 e-05$ & $0.87410801 e-05$ & $0.12829588 \mathrm{e}-04$ \\
\hline & $Z_{6}$ & $0.90972916 \mathrm{e}-09$ & $-0.10812602 e-08$ & $0.18991534 \mathrm{e}-08$ & $-0.20053974 e-08$ \\
\hline & $Z_{7}$ & $-0.58981537 e-04$ & $0.90918809 e-03$ & $-0.11452490 e-03$ & $0.78784157 e-03$ \\
\hline & $Z_{8}$ & $-0.12990752 e-07$ & $-0.26960555 e-04$ & $-0.11341777 e-06$ & $-0.18512345 e-04$ \\
\hline & $Z_{9}$ & $0.45872518 \mathrm{e}-08$ & $-0.36454880 e-06$ & $0.19903338 e-08$ & $-0.52896691 e-06$ \\
\hline \multicolumn{2}{|c|}{$\begin{array}{l}\text { Pressure range } \\
\text { Temperature range }\end{array}$} & $\begin{array}{c}0.001-110 \mathrm{MPa} \\
15-360^{\circ} \mathrm{C}\end{array}$ & $\begin{array}{c}0.001-20 \mathrm{MPa} \\
15-360^{\circ} \mathrm{C}\end{array}$ & $\begin{array}{c}0.001-20 \mathrm{MPa} \\
0.5-360^{\circ} \mathrm{C}\end{array}$ & $\begin{array}{c}0.001-20 \mathrm{MPa} \\
0.5-360^{\circ} \mathrm{C}\end{array}$ \\
\hline
\end{tabular}




\begin{tabular}{|c|c|c|c|c|c|}
\hline & & Liquid & Vapor & Liquid & Vapor \\
\hline \multirow{10}{*}{ 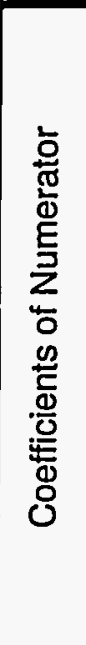 } & $Y_{0}$ & $0.10000000 e+01$ & $0.15089524 \mathrm{e}-05$ & $0.10000000 \mathrm{e}+01$ & $0.13299942 \mathrm{e}-04$ \\
\hline & $Y_{1}$ & $0.17472599 \mathrm{e}-01$ & $0.10000000 e+01$ & $-0.50430220 e-01$ & $0.10000000 e+01$ \\
\hline & $Y_{2}$ & $-0.20443098 \mathrm{e}-04$ & $-0.10000000 e+01$ & $0.12147449 e-02$ & $-0.10000000 e+01$ \\
\hline & $Y_{3}$ & $-0.17442012 e-06$ & $-0.16676705 e-02$ & $-0.29566543 e-04$ & $-0.56746973 e-02$ \\
\hline & $Y_{4}$ & $0.49564109 \mathrm{e}-02$ & $0.40111210 \mathrm{e}-07$ & $0.11719555 \mathrm{e}-01$ & $-0.32791354 e-06$ \\
\hline & $Y_{5}$ & $-0.40757664 e-04$ & $0.25625316 \mathrm{e}-10$ & $-0.10272834 e-03$ & $0.21636240 \mathrm{e}-08$ \\
\hline & $Y_{6}$ & $0.50676664 \mathrm{e}-07$ & $-0.40479650 e-12$ & $0.16483547 e-06$ & $-0.38485869 e-11$ \\
\hline & $Y_{7}$ & $0.50330978 e-04$ & $0.43379623 e-01$ & $0.74802254 e-03$ & $0.40896880 \mathrm{e}-01$ \\
\hline & $Y_{8}$ & $0.33914814 \mathrm{e}-06$ & $0.24991800 \mathrm{e}-02$ & $0.17552861 e-05$ & $0.27696827 \mathrm{e}-02$ \\
\hline & $Y_{9}$ & $-0.18383009 e-06$ & $-0.94755043 e-04$ & $-0.16978281 e-05$ & $-0.94741649 e-04$ \\
\hline \multirow{10}{*}{ 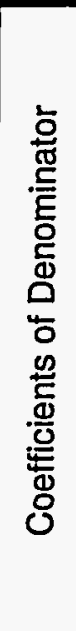 } & $Z_{0}$ & $0.10009476 e-02$ & $0.12636224 e+00$ & $0.10020170 e-02$ & $0.12789230 e+00$ \\
\hline & $Z_{1}$ & $0.16812589 \mathrm{e}-04$ & $-0.30463489 e+00$ & $-0.52711077 e-04$ & $-0.28996744 e+00$ \\
\hline & $Z_{2}$ & $-0.24582622 e-07$ & $0.27981880 \mathrm{e}-02$ & $0.14548166 e-05$ & $0.26873883 e-02$ \\
\hline & $Z_{3}$ & $-0.17014984 e-09$ & $0.51132337 e-05$ & $-0.36472636 e-07$ & $0.33783903 e-04$ \\
\hline & $Z_{4}$ & $0.48841156 e-05$ & $0.59318010 \mathrm{e}-02$ & $0.11718816 \mathrm{e}-04$ & $0.55690966 \mathrm{e}-02$ \\
\hline & $Z_{5}$ & $-0.32967985 e-07$ & $0.80972509 e-05$ & $-0.93182060 e-07$ & $0.72603809 e-05$ \\
\hline & $Z_{6}$ & $0.28619380 e-10$ & $-0.43798358 \mathrm{e}-07$ & $0.12768238 \mathrm{e}-09$ & $-0.44323127 e-07$ \\
\hline & $Z_{7}$ & $0.53249055 \mathrm{e}-07$ & $0.53046787 e-03$ & $0.72200359 \mathrm{e}-06$ & $0.49878874 \mathrm{e}-03$ \\
\hline & $Z_{8}$ & $0.30456698 \mathrm{e}-09$ & $-0.84916607 e-05$ & $0.18887078 \mathrm{e}-08$ & $-0.13186635 \mathrm{e}-04$ \\
\hline & $Z_{9}$ & $-0.12221899 e-09$ & $0.48444919 e-06$ & $-0.14167944 e-08$ & $0.72041771 e-06$ \\
\hline \multicolumn{2}{|c|}{$\begin{array}{l}\text { Pressure range } \\
\text { Temperature range }\end{array}$} & $\begin{array}{c}0.001-110 \mathrm{MPa} \\
15-360^{\circ} \mathrm{C}\end{array}$ & $\begin{array}{c}0.001-20 \mathrm{MPa} \\
15-360^{\circ} \mathrm{C}\end{array}$ & $\begin{array}{c}0.001-20 \mathrm{MPa} \\
0.5-360^{\circ} \mathrm{C}\end{array}$ & $\begin{array}{c}0.001-20 \mathrm{MPa} \\
0.5-360^{\circ} \mathrm{C}\end{array}$ \\
\hline
\end{tabular}


FEHMN MMS, ECD-22 LA-UR-94-3787, Rev. 1

Page 68 of 69

\begin{tabular}{|c|c|c|c|c|c|}
\hline & & 8 & & & \\
\hline & & Liquid & Vapor & Liquid & Vapor \\
\hline & $Y_{0}$ & $0.17409149 \mathrm{e}-02$ & $-0.13920783 e-03$ & $0.17395487 \mathrm{e}-02$ & $-0.67484241 e-04$ \\
\hline & $Y_{1}$ & $0.18894882 \mathrm{e}-04$ & $0.98434337 e-02$ & $0.18724784 \mathrm{e}-04$ & $0.36800173 e-02$ \\
\hline$\frac{2}{\frac{0}{\pi}}$ & $Y_{2}$ & $-0.66439332 e-07$ & $-0.51504232 \mathrm{e}-03$ & $-0.15981722 e-06$ & $0.10553076 \mathrm{e}-02$ \\
\hline$\stackrel{2}{\Phi}$ & $Y_{3}$ & $-0.23122388 \mathrm{e}-09$ & $0.62554603 e-04$ & $0.15081123 e-08$ & $0.75936247 e-04$ \\
\hline$\sum_{0}^{3}$ & $Y_{4}$ & $-0.31534914 e-05$ & $0.27105772 \mathrm{e}-04$ & $0.64958498 \mathrm{e}-04$ & $0.21890632 e-04$ \\
\hline$\stackrel{0}{\stackrel{0}{Z}}$ & $Y_{5}$ & $0.11120716 \mathrm{e}-07$ & $0.84981906 \mathrm{e}-05$ & $-0.31524525 e-06$ & $0.86065590 \mathrm{e}-05$ \\
\hline$\frac{\overline{\mathrm{D}}}{\mathrm{O}}$ & $Y_{6}$ & $-0.48576020 e-10$ & $0.34539757 e-07$ & $0.16965774 \mathrm{e}-09$ & $0.33613345 e-07$ \\
\hline "ॄ. & $Y_{7}$ & $0.28006861 e-07$ & $-0.25524682 e-03$ & $0.17392236 \mathrm{e}-05$ & $-0.18856602 e-03$ \\
\hline & $Y_{8}$ & $0.23225035 e-09$ & $0.00000000 e+00$ & $0.27900189 \mathrm{e}-08$ & $-0.44264826 e-05$ \\
\hline & $Y_{9}$ & $0.47180171 e-10$ & $0.12316788 \mathrm{e}-05$ & $-0.17278880 e-08$ & $0.64108570 \mathrm{e}-06$ \\
\hline & $Z_{0}$ & $0.10000000 \mathrm{e}+01$ & $0.10000000 e+01$ & $0.10000000 \mathrm{e}+01$ & $0.10000000 e+01$ \\
\hline & $Z_{1}$ & $0.10523153 e-01$ & $0.10000000 \mathrm{e}+01$ & $0.12149818 \mathrm{e}-01$ & $-0.10000000 e+01$ \\
\hline$\stackrel{\text { 윰 }}{=}$ & $Z_{2}$ & $-0.22658391 e-05$ & $-0.10000000 e+01$ & $-0.82786317 e-04$ & $-0.10000000 e+01$ \\
\hline $\bar{\varepsilon}$ & $Z_{3}$ & $-0.31796607 e-06$ & $-0.10000000 e+01$ & $0.90820763 e-06$ & $-0.16348067 e+00$ \\
\hline 㐫 & $Z_{4}$ & $0.29869141 e-01$ & $0.10000000 \mathrm{e}+01$ & $0.56642913 e-01$ & $0.10000000 e+01$ \\
\hline 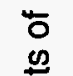 & $Z_{5}$ & $0.21844248 \mathrm{e}-03$ & $0.10000000 \mathrm{e}+01$ & $0.20657804 \mathrm{e}-02$ & $0.10000000 e+01$ \\
\hline . & $Z_{6}$ & $-0.87658855 e-06$ & $-0.22934622 e-03$ & $-0.86925068 \mathrm{e}-05$ & $\cdot-0.25908581 e-03$ \\
\hline 劳 & $Z_{7}$ & $0.41690362 e-03$ & $0.10000000 \mathrm{e}+01$ & $0.11471862 \mathrm{e}-02$ & $-0.10000000 e+01$ \\
\hline & $Z_{8}$ & $-0.25147022 e-05$ & $0.00000000 e+00$ & $0.00000000 \mathrm{e}+00$ & $0.00000000 e+00$ \\
\hline & $Z_{9}$ & $0.22144660 \mathrm{e}-05$ & $0.25834551 \mathrm{e}-01$ & $0.47391172 \mathrm{e}-04$ & $0.11181278 e-01$ \\
\hline $\begin{array}{r}\text { Pre } \\
\text { Temr }\end{array}$ & $\begin{array}{l}\text { range } \\
\text { e range }\end{array}$ & $\begin{array}{c}0.001-110 \mathrm{MPa} \\
0.001-360^{\circ} \mathrm{C}\end{array}$ & $\begin{array}{c}0.001-20 \mathrm{MPa} \\
15-360^{\circ} \mathrm{C}\end{array}$ & $\begin{array}{c}0.001-20 \mathrm{MPa} \\
0.5-360^{\circ} \mathrm{C}\end{array}$ & $\begin{array}{c}0.001-20 \mathrm{MPa} \\
0.5-360^{\circ} \mathrm{C}\end{array}$ \\
\hline
\end{tabular}

Modification date: $3 / 30 / 95$ 


\begin{tabular}{|c|c|c|c|}
\hline & & Pressure & Temperature \\
\hline \multirow{5}{*}{ 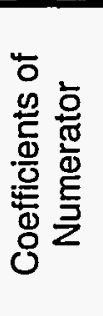 } & $Y_{0}$ & $0.71725602 \mathrm{e}-03$ & $-0.25048121 e-05$ \\
\hline & $Y_{1}$ & $0.22607516 e-04$ & $0.45249584 e-02$ \\
\hline & $Y_{2}$ & $0.26178556 \mathrm{e}-05$ & $0.33551528 e+00$ \\
\hline & $Y_{3}$ & $-0.10516335 e-07$ & $0.10000000 \mathrm{e}+01$ \\
\hline & $Y_{4}$ & $0.63167028 \mathrm{e}-09$ & $0.12254786 e+00$ \\
\hline \multirow{5}{*}{ 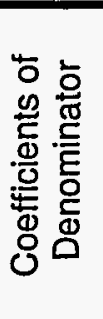 } & $Z_{0}$ & $0.10000000 \mathrm{e}+01$ & $0.20889841 e-06$ \\
\hline & $Z_{I}$ & $-0.22460012 e-02$ & $0.11587544 \mathrm{e}-03$ \\
\hline & $Z_{2}$ & $0.30234492 \mathrm{e}-05$ & $0.31934455 e-02$ \\
\hline & $Z_{3}$ & $-0.32466525 e-09$ & $0.45538151 \mathrm{e}-02$ \\
\hline & $Z_{4}$ & 0.0 & $0.23756593 e-03$ \\
\hline \multicolumn{2}{|c|}{$\begin{array}{c}\text { Pressure range } \\
\text { Temperature range }\end{array}$} & \multicolumn{2}{|c|}{$\begin{array}{c}0.00123-14.59410 \mathrm{MPa} \\
10-340^{\circ} \mathrm{C}\end{array}$} \\
\hline
\end{tabular}


